\title{
Über die festen Polyjodide der Alkalien, ihre Stabilität und Existenzbedingungen bei $25^{\circ}$.
}

\author{
Nach der Breslauer Dissertation von AnNa Hamberger, \\ mitgeteilt ron $R$. ABEGG. \\ Mit 3 Figuren im Text.
}

Die Tatsache, dafs Jod in Wasser sehr wenig löslich ist, in den wässerigen Lösungen seiner Metallsalze aber seine Löslichkeit aulserordentlich stark erhöht wird, ist durch die modernen physikalisch-chemischen Methoden aufgeklärt worden.

Es haben Le Blanc und Noyes ${ }^{1}$ aus Bestimmungen der Gefrierpunktserniedrigung wässeriger Jodkaliumlösungen vor und nach Zusatz von Jod geschlossen, dafs, da die Anzahl der erniedrigend wirkenden Moleküle in der Lösung sich durch Jodzusatz nicht merklich ändert, alles Jod chemisch als Polyjodid gebunden sein mufs. Welches Polyjodid nun vorliege, und ob sich überhaupt nur ein einziges oder verschiedene gebildet hatten, hat $\mathrm{J}_{\Delta \mathrm{K} O W \mathrm{wNN}^{2}}{ }^{2}$ für ziemlich verdünnte Lösungen durch Verteilungsversuche festgestellt: unter der Annahme, dais nur $\mathrm{KJ}_{\mathbf{8}}$ sich gebildet hat, erhielt er eine gute Konstante für die thermolytische Dissoziation von $\mathrm{KJ}_{3}$ in $\mathrm{KJ}+\mathrm{J}_{2}$, so dals man annehmen muls, dal's diese verdünnten Lösungen im wesentlichen $\mathrm{KJ}_{3}$ resp. dessen Anion $\mathrm{J}_{3}{ }^{\prime}$ enthalten.

Die in konzentrierteren Jodlösungen auftretende Änderung der Konstanten ist nach $J_{\Delta K}$ KowkIN durch die Bildung höherer Polyjodide zu erklären, und er weist mit Recht darauf hin, dafs, wenn höhere Polyjodide in konzentrierteren Lösungen auftreten, solche nach dem Massenwirkungsgesetz auch in schwachen Lösungen, wenn auch in geringem Mafse vorhanden sein müssen, da auch hier ihre Komponenten $\mathrm{KJ}_{3}$

1 Zeitschr. phys. Chem. 6, 401.

${ }^{2}$ Zeitschr. phys. Chem. 20, 36. 
und $J_{2}$ vorhanden sind. Julics MeYeR ${ }^{1}$ hat in ähnlicher Weise für die alkalischen Erden durch Gefrierpunktsbestimmungen bewiesen, dals Polyjodide in deren Lösung bestehen. Um sich auch über die Existenz fester Polyjodide zu orientieren, schmilzt er $\mathrm{J}$ und $\mathrm{Ca}_{2}$ in molekularen Verhältnissen zusammen und schliefst aus der Tensionsverringerung des Jods, auf das Vorhandensein der festen Verbindungen, obgleich die qualitative Tatsache der Erniedrigung der Tension keinen bindenden Beweis dafür enthält, dafs chemische Individuen vorliegen.

DAwson und GAWLER ${ }^{2}$ machten bei der Untersuchung der Verteilung von Jod zwischen einer wässerigen Jodkaliumlösung und Nitrobenzol, in dem KJ nicht löslich ist, die wichtige Beobachtung, dafs die jodhaltige Nitrobenzollösung Jodkalium löst und dadurch die Lösungsfähigkeit der wässerigen Lösung für Jod vermindert, und zwar soweit, bis fast das ganze $\mathrm{KJ}$ in die Nitrobenzolschicht gezogen worden ist. Man kann diese Tatsache auch so deuten, dafs sich im Wasser Polyjodide bilden, die im Nitrobenzol bedeutend löslicher sind als in Wasser, so dafs ihr Verteilungsgleichgewicht sebr zugunsten des Nitrobenzols liegt. Das Verhältnis $J_{2}: \mathrm{KJ}$ in Nitrobenzol nähert sich mit wachsender Jodkonzentration dem Werte 4, so dafs also in der Nitrobenzollösung die Bildung eines Polyjodids $\mathrm{KJ}_{9}$ anzunehmen wäre. Die Untersuchungen wurden von Dawson und Goodsox ${ }^{3}$ auf $\mathrm{NH}_{4}, \mathrm{Na}, \mathrm{Li}, \mathrm{Rb}$, Cs und Tetramethylammoniumjodid ausgedehnt und in allen Fällen das Enneajodid als höchste Stufe gefunden. Feste Polyjodide zu isolieren ist ihnen nicht gelungen.

Das Vorhandensein von Polyjodiden in Lösungen ist durch alle diese Versuche unzweideutig erwiesen, aber über ihre Existenz im festen Zustande ist noch nichts zu folgern. Dawson und Goonson betonen das am Schlufs ihrer Arbeit, indem sie sagen: „Es ist vielleicht nicht unnötig anzudeuten, dafs diese Versuche nur für das Vorhandensein ron Polyjodiden in der Lösung beweisend sind und mit deren Vorhandensein im festen Zustande nichts zu tun haben; die aus einer Lösung ausfallenden Körper sind nicht immer identisch mit den Hauptkomponenten der Lösung". In der Tat wird aus einer Lösung immer zuerst diejenige Molekelart in fester

1 Z. anorg. Chem. 30, 113.

2 Journ. Chem. Soc. 81 (1902), 524.

3 Journ. Chem. Soc. 1904, Juni. 
Form ausfallen, deren Sättigungskonzentration bei der Versuchstemperatur zuerst erreicht ist. Es handelt sich also bei der Frage um die Existenz fester Verbindungen im wesentlichen um eine Löslichkeitsfrage. Dals die in fester Form auftretenden Molekelarten in der Lösung oft nur eine äufserst geringe Konzentration besitzen, ihre Sättigung aber eher erreichen als andere Molekeln, die gemäls dem Massenwirkungsgesetz in erheblich höherer Konzentration vorhanden sind, beweist die Tatsache, dafs Boduandder und Fittia ${ }^{1}$ eine Anzahl Ammoniakkomplexsalze in fester Form erbalten haben, für deren Existenz in Lösungen es keine Andeutungen gibt, wie z. B $\mathrm{Ag}_{2}\left(\mathrm{NH}_{3}\right)_{3} \mathrm{Cl}_{2}$ und $\mathrm{Ag}\left(\mathrm{NH}_{3}\right)_{3} \mathrm{Cl}$, während in der Lösung $\mathrm{Ag}\left(\mathrm{NH}_{3}\right)_{2} \mathrm{Cl}$ festgestellt wurde. Ebenso haben $\mathrm{W}_{\text {ALDEN }}$ und CenTNERszWER ${ }^{2} \mathrm{KJ}\left(\mathrm{SO}_{2}\right)_{4}$ und $(\mathrm{KJ})_{4}\left(\mathrm{SO}_{2}\right)_{14}$ isolieren können, ohne ihre Existenz in der Lösung beweisen zu können, während andererseits $\mathrm{Fox}^{3}$ das Vorhandensein von $\mathrm{KJ} .\left(\mathrm{SO}_{2}\right)$ in der Lösung experimentell sicher gestellt hat, ohne den festen Körper erhalten zu können.

Von den Polyjodiden der Alkalien sind in fester Form bisher dargestellt worden von $\mathrm{JOHNSON}^{4} \mathrm{KJ}_{3}$, von WELLS und WHEELER ${ }^{5}$ $\mathrm{RbJ}_{3}, \mathrm{CsJ}_{3}, \mathrm{CsJ}_{5}$. Die Bemühungen dieser Forscher, $\mathrm{KJ}_{5}$ und $\mathrm{RbJ}_{5}$ darzustellen, verliefen stets erfolglos.

Über die festen Polyjodide organischer Basen existieren zahlreiche Arbeiten, die bis in das Jahr 1854 zurückreichen. Von ibnen seien die Arbeiten von Weltzien ${ }^{6}$, MÜLler ${ }^{7}$, Dafert ${ }^{8}$, Jörgensen ${ }^{9}$ und GEUTHER $^{10}$ erwähnt, denen sich neue Arbeiten von STRöMHоLM ${ }^{11}$ anschliefsen.

Alle diese Untersuchungen stimmen darin überein, dals sie den Typus $\mathrm{R}^{\mathrm{I}} \mathrm{J}_{9}$ als oberen Grenztypus für die Polyjodidbildung angeben, wenn es sich um einwertige Basen handelt, denen bei den zweiwertigen Basen der Typus $\mathrm{R}^{\mathrm{Ir}} \mathrm{J}_{18}$ entspricht. Und STröмноцM schliefst, dals in allen diesen das negative Radikal $J_{9}{ }^{\prime}$ enthalten sein muls.

1 Zeitsehr. phys. Chem. 39, 597.

${ }^{2}$ Zeitschr. phys. Chem. 42, 432.

${ }^{3}$ Zeitschr. phys. Chem. 41, 458.

* Journ. Chem. Soc. 1877, 249.

${ }^{5} Z$. anorg. Chem. 1, 85. 442; 2, 225.

6 Lieb. Ann. 91, 33; 99, 1.

- Lieb. Ann. 108, 5.

8 Monatsh. 4, 496.

${ }^{9}$ Journ. prakt. Chem., N. F., 2, 3, 14, 15.

10 Journ. prakt. Chem. 240, 63.

11 Journ. prakt. Chem., N. F., 67, 345. 
Wir werden im folgenden sehen, dafs auch die Alkalien demselben Grenztypus des Enneajodids zustreben, und dafs die Individualität der bisher beschriebenen Stoffe keineswegs einwandsfrei festgestellt ist.

Wie aus den Beobachtungen aller Forscher hervorgeht, die sich mit den festen Polyjodiden der Alkalien beschäftigt haben, hat man es hier mit dissoziablen festen Körpern zu tun. Wenn man also von neuem daran ging, ihre Existenzfähigkeit zu untersuchen, so mulste dies auf Grund der den Dissoziationserscheinungen fester Körper zugrunde liegenden Gesetze des heterogenen Gleichgewichtes geschehen.

Wir bestimmten die für die verschiedenen Individuen charakteristischen Dissoziationsspannungen des Jods in Gestalt der Konzentrationen, die das Jod in Benzol erreicht, da Benzol praktisch nichts ron den Salzen, sondern nur das Jod löst. STrömroum ${ }^{1}$ hat bereits bei einer analogen Untersuchung organischer Polyjodide diese Methode angewandt.

Entzieht man einem Polyjodid durch Benzol Jod, so entsteht ein niederes Polyjodid oder Monojodid neben dem ersten als Bodenkörper und es entsteht gemäls der Phasenregel eine konstante Jodkonzentration im Benzol, solange von denselben beiden Bodenkörpern noch etwas vorhanden ist. Bei weiterer Jodentziehung verschwindet schliefslich das höhere Polyjodid und die Jodkonzentration über dem allein zurückgebliebenen Bodenkörper wird variabel, bis eine neue und niedrigere Jodierungsstufe auftritt. Die Analyse der unter den variablen Jodkonzentrationen beständigen einheitlichen Bodenkörper lebrt die einzelnen Inàividuen kennen, die konstanten Dissoziationsdrucke ihre Existenzgrenzen und die Grölse der Bindungsfestigkeit des Jods in ihnen.

Statt des Abbaues kann man natürlich auch durch wachsende Jodkonzentrationen die Polyjodide aus den Monojodiden systematisch aufbauen.

Die hierauf basierte Versuchsmethode war im wesentlichen die folgende:

Um zunächst festzustellen, ob überhaupt bei der Versuchstemperatur von $25^{\circ}$ Polyjodide existieren, wurde das Monojodid mit einer an Jod nahezu gesättigten Benzollösung geschüttelt. Wir hatten dann über dem Monojodid den höchsten bei dieser Tempe-

1 Journ. prakt. Chem. 66 (1902), 423. 517; 67 (1903), 345. 
ratur erreichbaren Joddruck. Es war daher für alle Polyjodide, deren Dissoziationsdruck bei $25^{\circ}$ kleiner ist als der über reinem Jod, die Entstehungsmöglichkeit gegeben. Die Reaktion:

$$
\text { Monojodid }+ \text { Jod } \longleftrightarrow \text { Polyjodid }
$$

mulste von links nach rechts verlaufen. Indem Jod von dem Monojodid aufgenommen und Polyjodid gebildet wurde, sank die Jodkonzentration im Benzol und durch Hinzufügen immer neuer Mengen von Monojodid mulste schlielslich eine Jodkonzentration erreicht werden, eine "Jodtension", wie es im folgenden heifsen soll, die sich durch Hinzufügung von Monojodid nicht mehr ändert; und die für dasjenige Polyjodid charakteristisch ist, dessen Dissoziationsprodukt aufser Jod das Monojodid ist.

Durch weitere allmähliche Jodzusätze wurden die höheren Polyjodide aufgesucht, bis schliefslich die Lösung die Sättigungskonzentration für Jod zeigte; dann war Jod der zweite Bodenkörper neben dem höchsten erreichbaren Polyjodid. In den meisten Fällen wurde jedoch so verfahren, dals nicht Jod, sondern abgemessene Mengen von nahezu an Jod gesättigtem Benzol, dessen Jodgehalt durch Titration bekannt war, zum Monojodid hinzugefügt wurden, und zwar solange, bis diese Konzentration nach längerem Schütteln nicht mehr abnahm; man hatte dann das höchste Polyjodid allein als Bodenkörper. Der Jodgehalt der Bodenkörper konnte entweder, soweit ihre Unbeständigkeit dies erlaubte, durch Analyse oder durch Berechnen des aus dem Benzol aufgenommenen Jods bestimmt werden.

Um die Gleichgewichte ron der entgegengesetzten Seite zu erreichen, wurden die so erhaltenen höchsten Jodide zunächst mit Benzollösungen geschüttelt, deren Konzentration nur wenig unter der vorher ermittelten Gleichgewichtskonzentration für das höchste Jodid lag.

Diese mehrfach erneuerte Lösung wurde durch die Dissoziation des hohen Polyjodids immer wieder auf die Gleichgewichtskonzentration heraufgebracht, so lange noch hohes Polyjodid übrig war. Die Beibehaltung ihrer Jodkonzentration zeigte schliefslich an, dafs das hohe Polyjodid verschwunden und das nächst niedere Polyjodid allein am Boden lag, und man konnte sicher sein, dals wegen der hohen Konzentration der auslaugenden Lösung dieses niedere Polyjodid seinerseits noch kein Jod abgespalten hatte. Dieses Vorgehen stellt auch sicher, dafs alles durch die Analyse des Bodenkörpers 
bestimmte Jod chemisch gebunden also nicht mechanisch beigemengt ist; sonst hätte sich im Benzol Jodsättigung einstellen oder die Gleichgewichtskonzentration wenigstens deutlich überschritten werden müssen. Das konnte um so deutlicher erkannt werden, je weniger die gewählte Jodlösung unter der Gleichgewichtskonzentration lag, da dann nur wenig Jod zur Herstellung des Gleichgewichtes nötig war, dieses also um so leichter im Falle beigemengten Jods überschritten werden konnte.

Damit ist die Versuchsmethode im allgemeinen wiedergegeben, die Versuche konnten jedoch nicht immer genau nach diesem Schema von Anfang bis zu Ende durchgeführt, es mufsten immer erst orientierende Versuche gemacht werden. Aufserdem stellten sich beim Arbeiten eine Reihe von Schwierigkeiten ein; vor allem war es die starke Hygroskopie vieler Polyjodide, die das Arbeiten sehr un. angenehm machten und dazu zwangen, immer neue Versuche anzusetzen, ehe vollständig eindeutige Resultate erlangt wurden. Dazu kam, dals zwar die Gleichgewichte selbst sich von beiden Seiten meistens schnell und scharf einstellten, wenn auch keineswegs immer, dafs aber bei den Versuchen zur Herstellung der einheitlichen Bodenkörper trotz feiner Pulverung der Monojodide die Aufnahme der letzten Jodmengen aufserordentlich langsam erfolgte und oft mehrere Wochen ja Monate dauerte, so dafs der Jodtiter der Lösung über den Gleichgewichtsdruck stieg und sich scheinbar nicht mehr änderte, noch ehe die Polyjodidbildung völlig beendet war. Die festen Partikeln waren dann nur äufserlich in das höhere Polyjodid übergeführt, während das Eindringen in das innere wegen der Langsamkeit der Diffusion in festen Stoffen noch nicht vollendet war. Analysierte man in diesem Stadium den Bodenkörper, so fand man Gewichtsverhältnisse Monojodid: Jod, die zwischen zwei möglichen Polyjodiden lagen. Aufserdem waren die Analysenfehler in den meisten Fällen dadurch grols, dals beim Herausnehmen des Polyjodids aus den konzentrierten Benzollösungen das Benzol schnell verdunstete und sein Jod auf dem Bodenkörper hinterliefs.

Es sollen im folgenden natürlich nicht alle Versuche, die zu demselben Resultat geführt haben, wiedergegeben werden, sondern nur so viele, als zur Sicherstellung der Resultate nötig sind.

\section{Kaliumpolyjodide.}

Die Versuche wurden sämtlich bei $25^{\circ}$ durchgeführt und ein OsTwaLdscher Thermostat mit Schüttelvorrichtung benutzt, der 
durch einen Heifsluftmotor getrieben wurde. Die Reagentien waren sämtlich von KaHLbauM als rein bezogen, und für die vorliegende Untersuchung schien eine Prüfung auf ihre Reinheit nicht nötig. Zunächst wurde die Löslichleit von Jod in Benzol bei $25^{\circ}$ bestimmt, indem Benzol mit Jod geschüttelt wurde. Die gesättigte Lösung, in der Jod am Boden lag, wurde dann auf ca. $50^{\circ}$ erhitzt, mehrfach in der Hand geschüttelt und zur Abkühlung auf $25^{\circ}$ in den Thermostaten gehängt und nochmals titriert. Nach einigen Monaten wurde die Bestimmung noch einmal wiederholt und genügende Übereinstimmung aller Werte gefunden.

11 an Jod bei $25^{\circ}$ gesättigter Benzollösung enthält 1.095 g-Atome $\mathrm{J}=0.547 \mathrm{Mol} \mathrm{J}_{2}=139 \mathrm{~g} \mathrm{Jod}$.

An Jod ganz oder nabezu gesättigte Lösungen wurden nun mit KJ geschüttelt; schon nach einem Tage zeigte sich eine deutliche Abnahme des Jods im Benzol.

Tabelle 1.

Jodkaliumzusatz zu einer gesättigten Jodlösung.

\begin{tabular}{|c|c|c|c|}
\hline Datum & $\begin{array}{l}\text { Benzol } \\
\text { in eem }\end{array}$ & $\begin{array}{r}\mathrm{KJ} \\
\text { in } \mathrm{g} \\
\end{array}$ & $\begin{array}{l}\text { cem Thios. } 0.1 \mathrm{n} \text {. auf } \\
1 \text { ccm Lösung }\end{array}$ \\
\hline 15. Dez. & 275 & 3.71 & 10.95 \\
\hline 16. , & & & 9.55 \\
\hline 19. & & & 9.55 \\
\hline 22. " & & & 7.48 \\
\hline 29. , & & 27 & 7.46 \\
\hline 10. Jan. & & & 6.68 \\
\hline 15. , & & & 6.68 \\
\hline & & 1.65 & 6.68 \\
\hline 26. " & & & 6.68 \\
\hline 30. & & & 6.68 \\
\hline
\end{tabular}

Tabelle 1 zeigt die Durchführung eines solchen Versuches; sobald der Titer sich durch weiteres Schütteln nicht mehr änderte, wurden neue Jodkaliummengen dazugetan und das so lange fortgesetzt, bis Jodkaliumzusatz den Titer des Benzols nicht mehr änderte. Dann ist KJ sicher Bodenkörper neben dem Polyjodid, dessen Dissoziationsdruck durch den übriggebliebenen Jodgehalt des Benzols definiert ist, in diesem Falle:

$6.68 \mathrm{ccm} 0.1 \mathrm{n}$. Thiosulfat auf $1 \mathrm{ccm}$ der Lösung. 
Da reines Jod, wie aus der Löslichkeitsbestimmung herrorgeht, im Benzol einen Joddruck entsprechend $10.95 \mathrm{ccm} 0.1 \mathrm{n}$. Thiosulfatlösung hat, so beträgt also der Dissoziationsdruck des Polyjodids

$$
\frac{6.68}{10.95}=0.60 \text { des Joddrucks über reinem Jod. }
$$

Tabelle 2.

Gesättigte Jod-Benzollösung + Jodkalium

1 cem gesättigte Jod-Benzollösung verbraucht $10.95 \mathrm{ecm} 0.1 \mathrm{n}$. Thios.

\begin{tabular}{c|c|c|c|c|c}
\hline Nr. & $\begin{array}{c}\text { ecm gesättigtes } \\
\text { Jod-Benzol }\end{array}$ & $\begin{array}{c}\text { KJ } \\
\text { in } g\end{array}$ & $\begin{array}{c}\text { Tage des } \\
\text { Schüttelns }\end{array}$ & Endtiter & Mittelwert \\
\hdashline & 120 & 3.3 & 20 & 6.52 & \\
\hline 1 & 130 & 10.5 & 20 & 6.61 & \\
2 & 240 & 6.4 & 3 & 6.57 & $\mathbf{6 . 5 9}$ \\
3 & 275 & 8.1 & 44 & 6.68 & \\
4 & 280 & 4.8 & 26 & 6.60 & \\
5 & & & & &
\end{tabular}

In Tabelle 2 sina die Endresultate einer Reihe in gleicher Weise durchgeführter Versuche angeführt, die einen Mittelwert von 6.59 gaben.

Da zur Untersuchung des Jodtiters nur 1 oder $2 \mathrm{ccm}$ Benzol abpipettiert wurden, wenn es sich um konzentriertere Lösungen handelte, so können Abweichungen von 1\% als zulässig gelten; die Pipetten waren sämtlich auf Einfüllen geeicht und wurden zum Titrieren ausgespült, um Benetzungsfehler auszuschalten. Eine weitere Fehlerquelle liegt in dem grofsen Temperaturkoeffizienten der Jodlöslichkeit im Benzol, die bei Erniedrigung der Temperatur um $10^{\circ}$ auf die Hälfte zurückgeht.

Tabelle 3.

Jodzusatz zu Benzol und Jodkalium.

\begin{tabular}{|c|c|c|c|c|c|}
\hline Datum & $\begin{array}{l}\text { Benzol } \\
\text { in ecm }\end{array}$ & $\begin{array}{l}\mathrm{KJ} \\
\text { in } \mathrm{g}\end{array}$ & $\begin{array}{l}\text { Jod } \\
\text { in } g\end{array}$ & $\begin{array}{l}\text { cem Thios. } 0.1 \mathrm{n} \text {. } \\
\text { auf } 1 \mathrm{ccm} \text { Lösung }\end{array}$ & Bemerkungen \\
\hline 20. Febr. & 115 & 9.01 & 7.05 & 4.78 & \\
\hline 9. März & & & & 4.78 & Es ist noeh kein Jod \\
\hline 9.,$"$ & & & 4.21 & & $\begin{array}{l}\text { von KJ autgenommen } \\
\text { worden. }\end{array}$ \\
\hline 27. & & & & $6.57 *$ & * Der Titer hätte bis $\mathbf{7 . 4}$ \\
\hline $\begin{array}{l}\text { 27. " } " \\
\text { 8. April }\end{array}$ & & & 3.90 & 6.75 & $\begin{array}{l}\text { alles Jod in d. Jösung } \\
\text { geblieben wäre. }\end{array}$ \\
\hline 11. " & & & & 6.60 & \\
\hline
\end{tabular}


Tabelle 3 zeigt einen Versuch, der angestellt wurde, um das Gleichgewicht durch Jodzusatz von unten her, d. h. von niedrigeren Konzentrationen aus zu erreichen.

Alle Versuche nach dieser Richtung waren zuerst mifsglückt und hatten völlig unreproduzierbare Werte ergeben.

Schon bei geringen Jodzusätzen zu Benzol und Jodkalium ging nämlich Jod in den Bodenkörper, ohne dafs ein Gleichgewichtspunkt erreicht werden konnte. Beim Abgiefsen der Lösung zeigte sich am Boden eine zähe Flüssigkeit, es ergab sich, dals Feuchtigkeit die Ursache war. Da Benzol durch den Schliff der Stopfen leicht durchkriecht, wurden die Gummikappen, mit denen die Flaschen im Thermostaten gegen das Wasser geschützt wurden, durch herauskriechendes Jod zerstört und Spuren von Wasser drangen ein.

Der grofse Einflufs der Spuren von Wasser wurde durch die Löslichkeit von Jod in konzentrierten Jodkaliumlösungen und von Jodkalium in viel Jod enthaltenden Lösungen festgestellt, worüber weiter unten berichtet werden wird.

Jedenfalls ist klar, dafs, sobald auch nur Spuren von Wasser in die Flaschen kamen, sich eine gesättigte Jodkaliumlösung bilden mufste, die, wie längst bekannt, Jod in grofsen Mengen löst, also dem Benzol Jod auch bei niedrigerer als Gleichgewichtskonzentration entzieben mulste.

Wir benutzten daher weiterhin zugeschmolzene Flaschen und gut getrocknete Substanzen. Die früheren Versuche wurden sämtlich wiederholt. Versuch 1 und 2 von Tabelle 2 sind ebenfalls in zugeschmolzenen Flaschen gemacht, ebenso die von Tabelle 3 und 4 .

Tabelle 3 zeigt, dals bei dem Jodtiter 4.78 noch kein Jod vom Bodenkörper aufgenommen worden ist. Beim zweiten Jodzusatz bätte der Titer bis 7.4 steigen müssen; er geht aber auf die Gleichgewichtskonzentration 6.6 zurück und bleibt auch bei weiterem Jodzusatz konstant.

Tabelle 4.

Verschiedene Jodzusätze zu Benzol und Jodkalium zur Kontrolle des Gleichgewichtes.

\begin{tabular}{|c|c|c|c|c|c|}
\hline $\begin{array}{l}\text { Versuchs- } \\
\text { dauer } \\
\text { in Tage }\end{array}$ & $\begin{array}{l}\text { Benzol } \\
\text { in ecm }\end{array}$ & $\begin{array}{l}\mathrm{KJ} \\
\text { in } \mathrm{g}\end{array}$ & $\begin{array}{l}\text { Jod } \\
\text { in } \mathrm{g}\end{array}$ & $\begin{array}{l}\text { cem Thiosulfat } \\
0.1 \mathrm{n} \text {, auf } 1 \mathrm{cem} \\
\text { der Lösung }\end{array}$ & $\begin{array}{l}\text { cem Thios. pro cem } \\
\text { d. Lösg., falls d. Jod } \\
\text { in der Lösung wäre }\end{array}$ \\
\hline 8 & 120 & 10.5 & 38 & 6.73 & 24.98 \\
\hline 44 & 115 & 9.0 & 15 & 6.60 & 10.8 \\
\hline 24 & 235 & 2.7 & 32 & 6.70 & 10.7 \\
\hline
\end{tabular}


Tabelle 4 enthält Versuche, die zeigen, wie hoch der Titer hätte steigen müssen, falls alles Jod in der Lösung geblieben wäre. Der Titer 24.9 ist natürlich in Benzol nicht erreichbar und nur angegeben, um zu zeigen, wie stark Jod vom Bodenkörpen aufgenommen worden ist.

\section{Tabelle 5.}

Gleichgewichtsüberschreitung durch Jodzusatz zur Bestimmung des entstandenen Bodenkörpers.

\begin{tabular}{|c|c|c|c|c|c|c|c|c|}
\hline 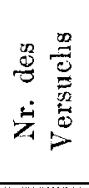 & 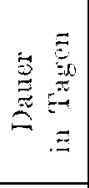 & 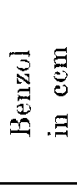 & 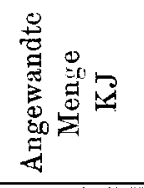 & 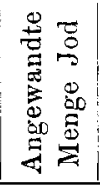 & 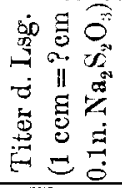 & 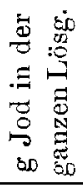 & 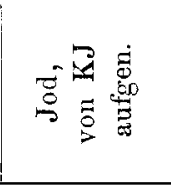 & 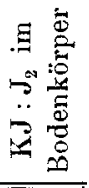 \\
\hline 1 & 50 & 120 & $\begin{array}{l}3.32 \mathrm{~g}= \\
20 \mathrm{mmol}^{1}\end{array}$ & $24.9 \mathrm{~g}$ & 7.04 & 10.3 & $\begin{array}{c}14.6 \mathrm{~g}= \\
57.6 \mathrm{mmol}^{1}\end{array}$ & $1: 2.9$ \\
\hline 2 & (1) & 135 & $\begin{array}{l}8.49 \mathrm{~g}= \\
51 \mathrm{mmol}\end{array}$ & 50.8 & 6.91 & 11.9 & $\begin{array}{c}38.9 \mathrm{~g}= \\
153 \mathrm{mmol}\end{array}$ & $1: 3$ \\
\hline
\end{tabular}

Die Versuche Tabelle 5 zeigen nun, dals das Gleichgewicht überschritten wird, wenn das Verhältnis der aufgenommenen Jodmenge $J_{2}: K J 1: 3$ ist, $d$. h. wenn sich das Heptajodid $\mathrm{KJ}_{7}$ gebildet hat.

Um dieses Resultat sicherzustellen, wurde ein neuer Versuch in der Weise angesetzt, dafs auf $\mathrm{KJ}_{7}$ berechnete Mengen von $J$ und $\mathrm{KJ}$ und zwar

$$
\begin{array}{r}
19.5 \mathrm{~g} \mathrm{~J}=77.0 \mathrm{Mol} \mathrm{J}_{2} \quad \text { und } \\
4.2 \mathrm{~g} \mathrm{KJ}=25.4 \mathrm{Mol}
\end{array}
$$

mit $60 \mathrm{ccm}$ einer an Jod ca. $80 \%$ gesättigten Lösung 2 Monate lang geschüttelt wurden. Hätte sich $\mathrm{KJ}_{3}$ oder $\mathrm{KJ}_{5}$ gebildet, so mufste das Benzol bei weitem Sättigungskonzentration erreichen. War aber $\mathrm{KJ}_{9}$ möglich, d. h. war bei den Versuchen von Tabelle 5, bei denen das Gleichgewicht ja nur wenig überschritten war, nur scheinbar die Jodaufnahme beendet, so mulsten den $60 \mathrm{ccm}$ Lösung $6 \mathrm{~g}$ Jod entzogen werden, also die Gleichgewichtskonzentration sich einstellen.

Die Titration der Lösung zeigte nun eine etwas höhere Konzentration, als die ursprüngliche, die bei Bildung von $\mathrm{KJ}_{7}$ sich hätte erhalten müssen, aber erstens lag diese noch deutlich unter 
der Sättigung und ihr Anwachsen konnte dadurch aufgeklärt werden, dafs in dem langen schmalen Hals der zugeschmolzenen Flasche sich etwas KJ zusammengeballt hatte, das hier der Einwirkung des Jods etwas entging, so dafs dies in die Lösung übergehen mulste. Eine Analyse der im Hals festgesetzten Substanz ergab $\mathrm{KJ}_{6.2}$, also wie nach obigem zu vermuten war, einen etwas zu kleinen Wert. Der in der Elasche gebliebene Hauptteil des Bodenkörpers wurde nun schnell auf Tonplatten abgeprefst und zwei gesonderte Portionen analysiert. Die eine Portion wurde in ein geschlossenes Wägegläschen gebracht, gewogen und dann bei ca. $60-80^{\circ}$ Jod im Trockenschrank abgedampft und das zurückbleibende Jodkalium zurückgewogen.

$$
\begin{aligned}
0.4957 \mathrm{~g} \text { Polyjodid enthielten } 0.4052 \mathrm{~g} \mathrm{~J} & =81.8 \% \\
\text { ber. für } \mathrm{KJ}_{7} & =82.1 \%
\end{aligned}
$$

Die zweite Portion wurde ungewogen mit ca. $100 \mathrm{ccm}$ Benzol in einen Scheidetrichter gespült und etwas Wasser dazu getan; dann geht das ganze KJ und ein kleiner Teil des Jods in die wässerige Schicht, die rom Benzol getrennt wurde. Durch mehrmaliges Hinzufügen neuer Portionen Wasser wurde alles KJ quantitativ aus dem Scheidetrichter in einen kleinen Rundkolben gespült. Die Benzolschicht wurde in einem Mefskolben auf $200 \mathrm{ccm}$ aufgefüllt.

Das in der Jodkaliumlösung befindliche Jod wurde titriert und dann das Jodion mit $\mathrm{AgNO}_{3}$ bestimmt. Von der Benzolschicht wurden je $10 \mathrm{ccm}$ titriert.

Es wurde mit $\mathrm{AgNO}_{3} 2.517$ äquiv. ${ }^{1} \mathrm{~J}_{2}$ festgestellt, davon ist abzuziehen 0.615 , die in der wässerigen Schicht durch die Thiosulfattitration aus freiem Jod entstanden waren. Es bleiben also

\section{$1.902 \mathrm{mmol} \mathrm{KJ}$.}

Die Benzolschicht enthielt $5.537 \mathrm{mmol} \mathrm{J}_{2}$; dazu kommen aus der wässerigen Schicht $0.307 \mathrm{mmol} J_{2}$, zusammen also $5.844 \mathrm{mmol}_{2}$.

Das Analysenresultat ist also $1.902 \mathrm{KJ}: 5.844 \mathrm{~J}_{2}=1: 3.07$; in Gewichtsprozenten: $82.5 \%$ Jod statt $82.1 \%$ für $\mathrm{KJ}_{7}$.

Mithin war $\mathrm{KJ}_{7}$ als Formel für den Bodenkörper festgelegt.

$9 \mathrm{~g}$ dieses Körpers wurden nun, wie aus Tabelle 6 ersichtlich ist, mit Jod-Benzollösungen von nur wenig niedrigerem als dem Gleichgewichtstiter geschüttelt, und zwar zunächst mit nur $20 \mathrm{ccm}$

1 äquiv. $=\frac{\ddot{\text { Aquivalent }}}{1000}$. 
einer Benzollösung vom Titer 5.5. Um diese $20 \mathrm{ccm}$ von $5 . \bar{s}$ auf den Gleichgewichtstiter von $6.6 \mathrm{zu}$ bringen, sind nur $0.27 \mathrm{~g} \mathrm{Jod}$ nötig; war also das durch die Analyse festgestellte Jod nicht chemisch als $\mathrm{KJ}_{7}$ gebunden, sondern vielleicht nur ein Gemisch von $\mathrm{KJ}_{5}$ und Jod, so mufsten die $9 \mathrm{~g}$ Bodenkörper $2.46 \mathrm{~g}$ freies Jod enthalten, und dies hätte in den $20 \mathrm{ccm}$ der zugegebenen Lösung Jodsättigung bewirkt.

Tabelle 6.

$9 \mathrm{~g} \mathrm{~K}_{7}$ mit Benzol.

\begin{tabular}{c|c|c|c|c}
\hline \multirow{2}{*}{$\begin{array}{c}\text { Dauer } \\
\text { in Tagen }\end{array}$} & Benzol & \multicolumn{3}{|c}{ Joditer } \\
in cem & vorhanden & ngehher \\
\hline 8 & 20 & 5.5 & 6.57 & \\
14 & 16 & 6.57 & 6.63 & \\
6 & 50 & 5.25 & 6.67 & \\
10 & 46 & 6.67 & 6.72 & Mittel: \\
4 & 50 & 5.25 & 6.72 & 6.68 \\
4 & 30 & 5.25 & 6.71 & \\
3 & 30 & 4.9 & 6.69 & \\
2 & 30 & 4.9 & 6.69 & \\
2 & 15 & 4.9 & 6.67 & \\
2 & 15 & 4.9 & 6.69 & \\
3 & 10 & 0 & 5.7 & \\
5 & 3 & 0 & 4 & \\
2 & 3 & 0 & 3.1 &
\end{tabular}

Die Tabelle beweist nun, dafs das nicht der Fall war, nach 8 Tagen war das Gleichgewicht praktisch erreicht und nach 6 Tagen weiteren Schüttelns nicht überschritten; wiederholte Zusätze solcher Lösungen, nachdem die alten abgegossen waren, führten immer zu nahezu demselben Titer, dessen Mittelwert 6.68 mit dem früher gefundenen innerhalb der Versuchsfehler übereinstimmt, bei der Jodtension 6.68 ist also $\mathrm{KJ}_{7}$ Bodenkörper neben $\mathrm{KJ}$.

Dal's der andere Bodenkörper bei dieser Konzentration $\mathrm{KJ}$ ist, wie alle früheren Versuche schon gezeigt hatten, wurde noch zweimal dadurch festgestellt, dals von den Lösungen, in denen sich der Gleichgewichtsdruck eingestellt hatte, $30 \mathrm{ccm}$ mit ca. $7 \mathrm{~g} \mathrm{KJ}$ einige Tage geschüttelt wurden: der Titer der Lösung änderte sich nicht im geringsten.

Polyjodide des Kaliums, deren Jodtension kleiner als $\mathrm{KJ}_{7}: \mathrm{KJ}$ ist, existieren also bei $25^{0}$ nicht. Die Möglichkeit ihrer Existenz- 
fähigkeit bei anderen Temperaturen ist damit keineswegs ausgeschlossen, wie die analogen Erfahrungen bei Salzhydraten lehren.

Die Angaben von $\mathrm{JOHNSON}^{1}$ über ein vermeintliches $\mathrm{KJ}_{3}$ werden weiter unten kritisiert werden.

\section{Lithium- und Natrium-Polyjodide.}

In gleicher Weise, wie beim Kalium wurden die Versuche mit LiJ und NaJ durchgeführt, indem je zwei Flaschen mit nahezu an Jod gesättigtem Benzol mit verschiedenen Mengen LiJ resp. NaJ geschüttelt und von Zeit zu Zeit titriert wurden.

Wie Tabelle 7 zeigt, war in beiden Fällen auch nach 4 Wochen keine Abnahme des Jodtiters zu bemerken. Es sind also bei $25^{\circ}$ weder Li- noch Na-Polyjodide in fester Form existenzfähig, d. h. die Tension, mit der sie ihr Jod abgäben, wenn sie existierten, wäre bei dieser Temperatur schon gröfser, als die über reinem Jod.

Tabelle 7.

NaJ- und LiJ-Zusatz zu gesättigter Jod-Benzollösung.

\begin{tabular}{|c|c|c|c|c|}
\hline $\mathrm{Nr}$. & $\begin{array}{c}\text { Dauer } \\
\text { in Tagen }\end{array}$ & $\begin{array}{l}\mathrm{NaJ} \text { resp. } \mathrm{LiJ} \\
\text { in } \mathrm{g}\end{array}$ & $\begin{array}{l}\text { Benzol } \\
\text { in ecm }\end{array}$ & $\begin{array}{l}\text { ecm Thios. } 0.1 \mathrm{n} \text {. } \\
\text { auf } 1 \text { cem Lösung }\end{array}$ \\
\hline 1 & $\begin{array}{r}0 \\
12 \\
27\end{array}$ & $\begin{array}{l}7.24 \\
\mathrm{NaJ}\end{array}$ & 70 & $\begin{array}{l}10.63 \\
10.63 \\
10.63\end{array}$ \\
\hline 2 & $\begin{array}{r}0 \\
12 \\
27\end{array}$ & $\begin{array}{l}1.95 \\
\mathrm{NaJ}\end{array}$ & 80 & $\begin{array}{l}10.63 \\
10.67 \\
10.50\end{array}$ \\
\hline 3 & $\begin{array}{r}0 \\
6 \\
12 \\
27\end{array}$ & $\begin{array}{c}3.006 \\
\mathrm{LiJ}\end{array}$ & 75 & $\begin{array}{l}10.60 \\
10.60 \\
10.62 \\
10.62\end{array}$ \\
\hline 4 & $\begin{array}{r}0 \\
6 \\
12 \\
27\end{array}$ & $\begin{array}{c}6.20 \\
\mathrm{LiJ}\end{array}$ & 75 & $\begin{array}{l}10.60 \\
10.67 \\
10.52 \\
10.69\end{array}$ \\
\hline
\end{tabular}

Ammonium-Polyjodide.

Wegen seiner vielfachen Ähnlichkeit mit den Alkalien wurde auch Ammonium auf seine Polyjodidbildung untersucht.

${ }^{1}$ Journ. Chem. Soc. 1879. 
Es wurde wieder gesättigte Jodlösung mit trockenem, reinem Ammoniumjodid geschüttelt, und es konnte starke Abnahme des Jodtiters im Benzol festgestellt werden. Wie Tabelle 8 zeigt, geht der Titer von 10.90 bis auf 0.58 zurück. Die Einstellungsgeschwindigkeit des Gleichgewichtes ist hier, wie übrigens in allen Fällen, deutlich von der angewandten Menge Monojodid abhängig, also von der mit dem Benzol in Berührung kommenden Oberfläche, und von dem Abstande der Jodtension im Benzol rom Gleichgewicht.

Tabelle 8.

$\mathrm{NH}_{4} \mathrm{~J}$-Zusatz zu verschieden starken Jod-Benzollösungen.

\begin{tabular}{r|c|c|c|c}
\hline $\begin{array}{c}\text { Dauer } \\
\text { in Tagen }\end{array}$ & $\begin{array}{c}\mathrm{NH}_{4} \mathrm{~J} \\
\text { in } \mathrm{g}\end{array}$ & $\begin{array}{c}\text { Benzol } \\
\text { in ecm }\end{array}$ & \multicolumn{2}{|c}{ Jodtiter } \\
& & & & \\
vorher & nachher \\
6 & 6.06 & 90 & 10.9 & 5.60 \\
9 & $6.06+1.56$ & 90 & 5.60 & 0.60 \\
13 & $6.06+1.56$ & 90 & 0.60 & $\mathbf{0 . 5 8}$ \\
\hline 8 & 1.0 & 50 & 19.0 & 1.60 \\
22 & $1.0+0.2$ & 50 & 1.60 & 0.95 \\
26 & $1.0+0.2+1.8$ & 50 & 0.95 & 0.60 \\
50 & $1.0+0.2+1.8$ & 50 & 0.60 & $\mathbf{0 . 5 8}$ \\
\hline
\end{tabular}

Gleichgewichtstension $=0.58$

Im Versuch 1 (Tabelle 8) war bei Anwendung von $6 \mathrm{~g} \mathrm{NH}_{4} J$ und einer gesättigten Lösung nach 6 Tagen schon die Hälfte Jod in das Monojodid gegangen und nach 9 Tagen das Gleichgewicht erreicht. In Versuch 2, wo nur $1 \mathrm{~g} \mathrm{NH}_{4} \mathrm{~J}$ angewendet wurde, und nur eine 1.90 starke Lösung benutzt wurde, ist nach 8 Tagen nur $1 / 5$ des Jods in den Bodenkörper übergegangen und erst nach 26 'Tagen wird das Gleichgewicht erreicht. Frische noch nicht jodierte Oberfächen beschleunigen natürlich ebenfalls die Reaktion.

Bei dem Jodtiter von 0.58 ist also Monojodid Bodenkörper neben einem Polyjodid, dessen Formel weiter festzustellen war. Dazu wurden drei Parallelversuche angesetzt (Tabelle 9), davon zwei mit gesättigten Jodlösungen aber verschiedenen Mengen Monojodid, während der dritte gleiche Mengen Monojodid wie der erste enthielt, aber eine nur halbgesättigte Jodlösung. Sie wurden bis zur Konstanz des Jodtiters geschüttelt.

Die Mengen waren so gewählt, dals in zwei Flaschen (Versuch 1 und 2) genug Jod zur Bildung von Trijodid war, der Titer 
aber über dem zuerst gefundenen Gleichgewicht bleiben mufste, so dafs man berechnen konnte, ob die zum Trijodid nötigen Mengen vom Bodenkörper aufgenommen waren, während der dritte Versuch einen eventuell vorhandenen höheren Gleichgewichtspunkt zeigen sollte.

Tabelle 9.

Beweis der Bildung von $\mathrm{NH}_{4} \mathrm{~J}_{3}$.

\begin{tabular}{|c|c|c|c|c|}
\hline Dauer & Benzol & & & m-atom $J_{1}$ vom \\
\hline in Tagen & in $\mathrm{ecm}$ & vorher & nachher & Bodenkörper aufgen. \\
\hline
\end{tabular}

I. Am Boden $3.238 \mathrm{~g}=22.1 \mathrm{mmol} \mathrm{NH}_{4} \mathrm{~J}$ :

\begin{tabular}{r|c|c|c|c}
8 & 50 & 9.61 & 7.09 & 12.6 \\
22 & 46 & 7.09 & 3.36 & 17.17 \\
28 & 42 & 3.36 & 2.76 & 2.50 \\
100 & 40 & 2.76 & 0.76 & 8.05 \\
\hline & \multicolumn{3}{|l}{$\mathbf{N H}_{4} \mathrm{~J}: \mathbf{J}_{2}=22.1: 20.2=1: 0.91}$.
\end{tabular}

II. $A m$ Boden $1.16 \mathrm{~g}=8.0 \mathrm{mmol} \mathrm{NH}_{4} \mathrm{~J}$ :

\begin{tabular}{r|c|c|c|r}
8 & 50 & 4.80 & 3.88 & 4.60 \\
22 & 46 & 3.88 & 2.61 & 5.80 \\
28 & 42 & 2.61 & 2.28 & 1.55 \\
100 & 38 & 2.28 & 1.15 & 4.25 \\
\cline { 2 - 3 } & \multicolumn{3}{|c}{$\mathrm{NH}_{\mathbf{4}} \mathrm{J}: \mathrm{J}_{2}=8.0: 16.2=1: 1.01}$.
\end{tabular}

III. Am Boden $1.264 \mathrm{~g}=8.7 \mathrm{mmol} \mathrm{NH} \mathrm{N}_{4} \mathrm{~J}$ :

\begin{tabular}{r|c|c|c|r}
8 & 50 & 9.61 & 9.02 & 2.85 \\
22 & 46 & 9.02 & 6.90 & 9.70 \\
28 & 42 & 6.90 & 6.64 & 1.20 \\
34 & 38 & 6.64 & 6.15 & 1.90 \\
100 & 32 & 6.15 & 5.88 & 0.80 \\
\hline \multicolumn{3}{|c}{$\mathrm{NH}_{4} J: J_{2}=8.7: 8.23=1: 0.94}$.
\end{tabular}

Aus den Versuchen Tabelle 9 geht hervor, dafs sich Trijodid gebildet hat; die aufgenommene Jodmenge ist unter Berücksichtigung der zum Titrieren entnommenen Mengen von je $4 \mathrm{cem}$ berechnet. Die Reaktion verläuft wieder äulserst langsam. Nach 28 Tagen war sie in keinem der drei Fälle beendigt; da nach 34 Tagen noch eine deutliche Jodabnahme zu konstatieren war, wurde noch 2 Monate länger geschüttelt, dann aber, da mit so verschiedenen Mengenverhältnissen derselbe Grenzwert, nämlich $\mathrm{NH}_{4} \mathrm{~J}_{3}$, erreicht 
worden war, durfte die Bildung ron $\mathrm{NH}_{4} \mathrm{~J}_{3}$ als erwiesen betrachtet werden.

Um den Dissoziationsdruck des $\mathrm{NH}_{4} \mathrm{~J}_{3}$ auch von oben her zu kontrollieren, und um nochmals zu prüfen, ob ein höheres Polyjodid als $\mathrm{NH}_{4} \mathrm{~J}_{3}$ existiert, stellten wir nach den Angaben von JoHNSON ${ }^{2} \mathrm{NH}_{4} \mathrm{~J}_{3}$ dar, indem man äquivalente Mengen $\mathrm{J}_{2}$ und $\mathrm{NH}_{4} \mathrm{~J}$ in wasserhaltigem Alkohol in der Hitze löste und stark abkühlte. Zwei Analysen der erhaltenen Kristalle durch Titration des Jods mit Thiosulfat in einer abgewogenen Menge der Substanz, die genau miteinander übereinstimmten, ergaben: $63 \% \mathrm{~J}$ statt $63.7 \%$. Sehr schöne Kristalle und bessere Analysen erhielt man, wenn man eine in der Wärme gesättigte Jodlösung mit annähernd der berechneten Menge $\mathrm{NH}_{4} \mathrm{~J}$ erwärmte und langsam auf dem erkaltenden Wasserbade abkühlen liels. Nach mehreren Stunden schieden sich schöne, grofse, blauschwarze Kristalle ab, deren optische Untersuchung auf das rhombische System schliefsen liefs; eine genaue Untersuchung war nicht möglich, da die Kristalle unter dem Mikroskop ihr Jod verloren und sich vollständig veränderten. Mehrere Analysen ergaben ibbereinstimmend: $64 \%$ Jod statt theor. $63.7 \%$. Von diesem $\mathrm{NH}_{4} \mathrm{~J}_{3}$ wurden dann einerseits, um seine Jodtension festzustellen, mehrere Portionen mit reinem Benzol einige Tage geschüttelt, wieder Benzol zugesetzt und geschüttelt; die erbaltenen Werte 0.55 und 0.56 (mit 0.01 n. $\mathrm{Na}_{2} \mathrm{~S}_{2} \mathrm{O}_{3}$ titriert) stimmten mit dem früher gefundenen 0.58 genügend überein. Ferner wurde in mehreren Versuchen $\mathrm{NH}_{4} J_{3}$ mit jodgesättigter Benzollösung längere Zeit geschüttelt, ohne dals eine Jodabnahme zu bemerken war. Spuren von Wasser verderben auch diese Versuche, weil $\mathrm{NH}_{4} \mathrm{~J}_{3}$ durch Wasser leicht zersetzlich ist; es wurden deshalb auch hier zugeschmolzene Flaschen angewendet.

Von Ammonium ist also nach diesen Versuchen bei $25^{0}$ nur $\mathrm{NH}_{\mathbf{4}} J_{3}$ beständig, und sein relativer Joddruck ist bei dieser Temperatur $\frac{0.50}{10.94}=0.053$ desjenigen des reinen $J_{0}$ ds bei $25^{\circ}$.

Für die Flaschen wurde nach vielem Ausprobieren schliefslich nebenstehende Form gewählt, die folgenden Bedingungen entsprach: Die Flasche mufs für wiederholtes Abschmelzen und Öffnen einen langen Hals haben; dieser ist vor Verstopfung durch den Bodenkörper zu schützen. 
Der bis auf den Boden reichende Einsatz wurde jedem Flaschenhals eingepalst und bestand aus einem dünwandigen, unten geschlossenen Röhrchen, das oben zugespitzt war und beim Öffnen der Flasche leicht herausgezogen werden konnte.

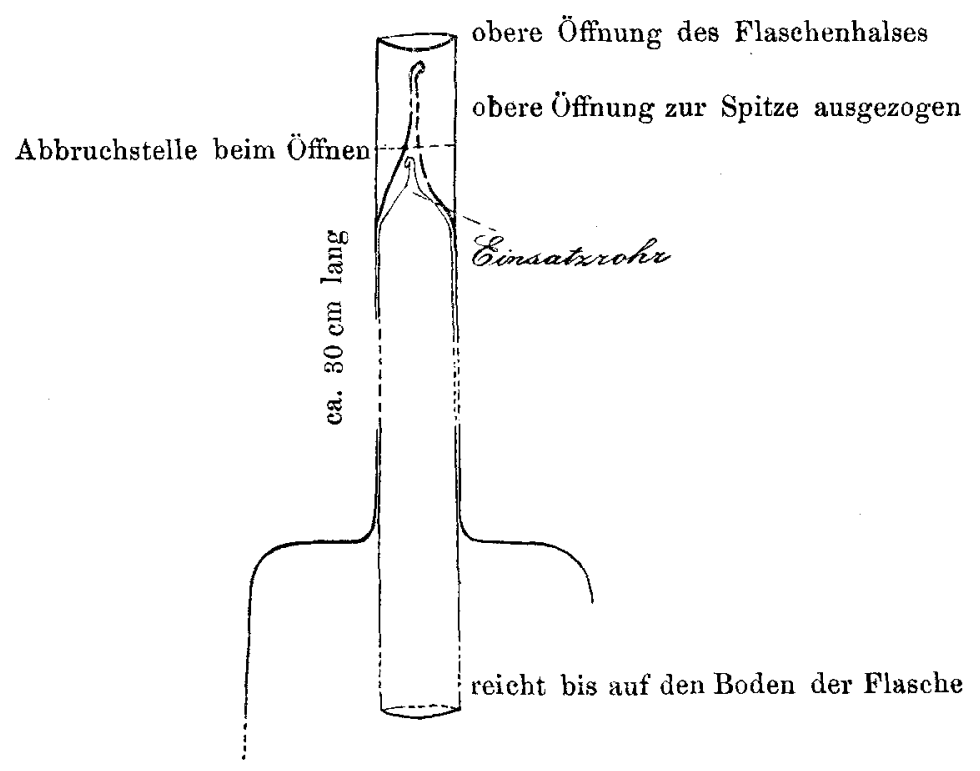

Fig. 1.

Rubidium-Polyjodide.

$\mathrm{RbJ}_{3}$ wurde nach den Angaben vun WeuLs ${ }^{1}$ durch Auflösen von $5.5 \mathrm{~g} \mathrm{RbJ}$ auf je $6 \mathrm{~g}$ Jod in wenig Wasser in der Hitze dargestellt. Beim Abkühlen auf Zimmertemperatur schieden sich, wie das auch WELLs beobachtet hat, stufenförmig angeordnete rhombische (also wobl mit $\mathrm{NH}_{4} \mathrm{~J}_{3}$ isomorphe) Kristalle ab, die bei der Analyse 53.6\% Jod statt 54.5\% ergaben. Unter dem Mikroskop waren Spuren von quadratischen Würfeln zu erkennen, die Jod einschlossen und wohl aus RbJ bestanden.

Es wurde nun einerseits $\mathrm{RbJ}_{3}$ mit reinem Benzol, andererseits jodgesättigte Benzollösungen mit grolsen Mengen RbJ geschüttelt. Tabelle 10 enthält die gefundenen Werte, die zeigen, dafs man auf beiden Wegen zu demselben Wert für die Jodtension von $\mathrm{RbJ}_{3}$ gelangt. Sie beträgt, wie die Tabelle zeigt, 0.265 , ist also nur halb so grofs als die von $\mathrm{NH}_{\mathbf{4}} \mathrm{J}_{3}$.

1 Z. anorg. Chem. 1, 442. 
Tabelle 10.

Jod-Tension von $\mathrm{RbJ}_{3}$ gegen $\mathrm{RbJ}$.

\begin{tabular}{|c|c|c|c|c|}
\hline $\begin{array}{c}\text { Dauer } \\
\text { in Tagen }\end{array}$ & $\begin{array}{l}\text { Angew. Menge } \\
\text { Bodenkörper in g }\end{array}$ & $\begin{array}{l}\text { Benzol } \\
\text { in com }\end{array}$ & $\begin{array}{l}\text { cem Thios } \\
\text { bei Beginn }\end{array}$ & $\begin{array}{l}\text { auf } 1 \mathrm{ccm} \\
\text { am Ende }\end{array}$ \\
\hline 14 & $8.00 \mathrm{RbJ}$ & 65 & 10.8 & $0.27^{1}$ \\
\hline 20 & 6.00 & 30 & 10.9 & 0.25 \\
\hline 30 & 0.78, & 65 & 0.85 & 0.266 \\
\hline 34 & 3.2 , & 45 & 3.15 & 0.27 \\
\hline 14 & $1.6 \mathrm{RbJ}_{3}$ & 15 & 0.0 & 0.267 \\
\hline 10 & $6.2 \quad$ & 15 & 0.0 & 0.26 \\
\hline
\end{tabular}

1 Titriert mit $0.01 \mathrm{n}$. Thiosulfat.

Mittel : 0.265

Bei einem weiteren Versuch nahmen $4.13 \mathrm{~g}=19.5 \mathrm{Mol} \mathrm{RbJ}$ ca. $4.4 \mathrm{~g}=35$ Atom Jod, $\mathrm{RbJ}_{3}$ entsprechend, auf.

Gerade beim Rubidium war die Realition so langsam, dafs oft mehrere Tage konstant gebliebene Werte sich schliefslich doch änderten, und viele Kontrollversuche nötig machten. Es kam hier vor, dafs nach zwei Monaten die Jodaufnahme aufbörte, ohne eine vollständige Umwandlung in das Polyjodid bewirkt zu haben. Diese wurde erst erzielt, indem die Polyjodidklumpen mehrmals zerrieben und von neuem mit Jod geschüttelt wurden. Daran lag es, dals die Versuche hier weniger systematisch durchgeführt werden konnten.

Bei gesteigertem Jodzusatz zu $\mathrm{RbJ}_{3}$ ergab sich eine sehr gut ausgeprägte, immer wiederkehrende neue Jodtension dem Titer 6.90 entsprechend; die bei dieser Tension aufgenommene Jodmenge betrug stets mehr als ein $\mathrm{Mol} \mathrm{J}_{2}$ pro $\mathrm{Mol} \mathrm{RbJ}_{3}$, so dafs die Bildung von $\mathrm{RbJ}_{7}$ unter Überspringung von $\mathrm{RbJ}_{5}$ wahrscheinlich war.

Bei drei rerschiedenen anderen Versuchen ergaben sich die Jodtensionen:

$$
6.926 .916 .80, \text { Mittel: } 6.88 \text {. }
$$

Bei weiterem Jodzusatz steigt dann der Titer und macht wieder Halt bei den Werten:

\section{$7.97 \quad 7.76 \quad 7.86, \quad$ Mittel: 7.89 .}

Die Bodenkörper unter diesen Jodtensionen wurden mehrfach analysiert und ergaben Werte zwischen $\mathrm{RbJ}_{7}$ und $\mathrm{RbJ}_{9}$, so dafs 
die Jodtension 7.89 dem Gleichgewichte $\mathrm{RbJ}_{7}: \mathrm{RbJ}_{9}$ entspricht. Die weitere Jodaufnahme dex analysierten Körper entsprach der Bildung von $\mathrm{RbJ}_{9}$.

Beim Abbau des so erhaltenen $\mathrm{RbJ}_{9}$ mit Benzol wurde das erste Mol Jod bei den Tensionen entsprechend den Titern

\section{$7.85 \quad 8.20 \quad 7.88, \quad$ Mittel: 7.96}

abgegeben.

Die Werte stimmen schlechter überein, als in den anderen untersuchten Fällen. Dies wird sicherlich durch die erwähnte grofse Trägheit ihrer Einstellung herbeigeführt, trotzdem sie sich deutlich als Haltepunkte bemerkbar machten, auch die nächste Tension blieb beim Abbau mit wiederholten Benzolzusätzen beim Titer 6.97 konstant, was mit dem früher erhaltenen Wert 6.88 genügend übereinstimmt. Dals diese zweite Tension dem Übergang von $\mathrm{RbJ}_{7}$ in $\mathrm{RbJ}_{3}$ entspricht, war nach allen bisher gemachten Erfahrungen $\mathrm{zu}$ vermuten; genaue Berechnungen waren hier deswegen nicht anzustellen, weil diese Versuche Monate lang gedauert batten, wodurch wiederholtes Umfüllen in neue Flaschen nötig war, sobald nämlich die Hälse zu kurz geworden waren.

Es schien daher nötig, noch einen ausschlaggebenden Versuch zur Prüfung obiger Annahmen anzustellen. $\mathrm{Zu}$ diesem $\mathrm{Z}$ wecke wurden auf $R b J_{5}$ resp. $R b J_{7}$ resp. $R b J_{9}$ berechnete Mengen von $R b J$ und Jod in geschlossenen Röhren zusammengeschmolzen, die Röhren zertrümmert und der fein gepulverte Inhalt mit Benzol geschüttelt, nachdem Analysen verschiedener Portionen gleichmälsige Durchmischung der Schmelzen angezeigt hatten. Es stellten sich nach genügend langem Schütteln in den Flaschen mit $\mathrm{RbJ}_{5}$ und $\mathrm{RbJ}_{7}$ die Titer 6.95 resp. 6.93 ein, was im Einklang mit den früheren Resultaten beweist, dals $\mathrm{RbJ}_{3}$ ohne Bildung von $R b J_{5}$ in $R b J_{7}$ übergeht, also $R b J_{5}$ bei $25^{0}$ richt existiert. $\mathrm{RbJ}_{9}$ zeigt die Tension entsprechend dem Titer 8.18.

Der Mittelwert der Titer aus sämtlichen Bestimmungen ist somit für $\mathrm{RbJ}_{7}: \mathrm{RbJ}_{3} 6.95$ und für $\mathrm{RbJ} J_{9}: \mathrm{RbJ}_{7}$ 8.01, entsprechend den relativen Jodtensionen 0.63 und 0.73 .

Bemerkenswert war bei diesen letzten Versuchen, dals sich nicht sofort die Gleichgewichtskonzentration im Benzol einstellte, sondern erst eine bedeutend höhere, die dann bei mehrtägigem Schütteln auf die oben angegebenen Werte zurückging and nun konstant blieb. Dies beweist, dafs die erstarten Schmelzen ron 
Monojodid und Jod, woraus die Bodenkörper bestanden, die beiden Komponenten wesentlich noch unverbunden enthalten haben, offenbar weil bei der Schmelztemperatur das Gleichgewicht einer fast vollständigen Dissoziation entspricht, die sich auch in der Kälte noch einige Zeit erhält. Erst allmählich stellt sich der einer viel geringeren Dissoziation entsprechende kalte Gleichgewichtszustand her, wie das auch $S_{A C K U R}{ }^{1}$ bei den $\mathrm{Cu}-\mathrm{Zn}$-Legierungen beobachtete. Die allgemeine Langsamkeit der Reaktionen fester Stoffe ist also auch für ihre Dissoziation malsgebend. ${ }^{2}$

\section{Cäsiam-Polyjodide.}

Aus dem Cäsiumjodid, das KaHubaum speziell für uns rein hergestellt hatte, wurde nach den Angaben von WeLLs ${ }^{3}$ Trijodid in sehr beständigen, prachtvollen Kristallen gewonnen; ferner das Pentajodid, das aber keine so guten Kristalle und auch etwas schwankende Analysen lieferte, wie das auch WeLLs angibt. Beide Körper wurden mit Benzol geschüttelt und bei $\mathrm{CsJ}_{3}$ der minimale Jodtiter von 0.0338 festgestellt. Es gelang, denselben Druck auch durch Schütteln von $C_{s} J$ mit einer Benzollösung vom Titer $2.78 \mathrm{zu}$ erbalten.

Sowohl $\mathrm{CsJ}_{3}$ wie $\mathrm{CsJ}_{5}$ wurden mit gesättigten Jod-Benzollösungen behandelt, wobei eine Jodaufnahme entsprechend der Bildung $\mathrm{CsJ}_{9}$ festgestellt wurde, wie die folgenden Tabellen zeigen.

Tabelle 11.

Jodaufnahme durch $\mathrm{Cs}_{5} \mathrm{~J}_{5}$.

Am Boden $4.078 \mathrm{~g}=6 \mathrm{mmol} \mathrm{CsJ}_{5}$.

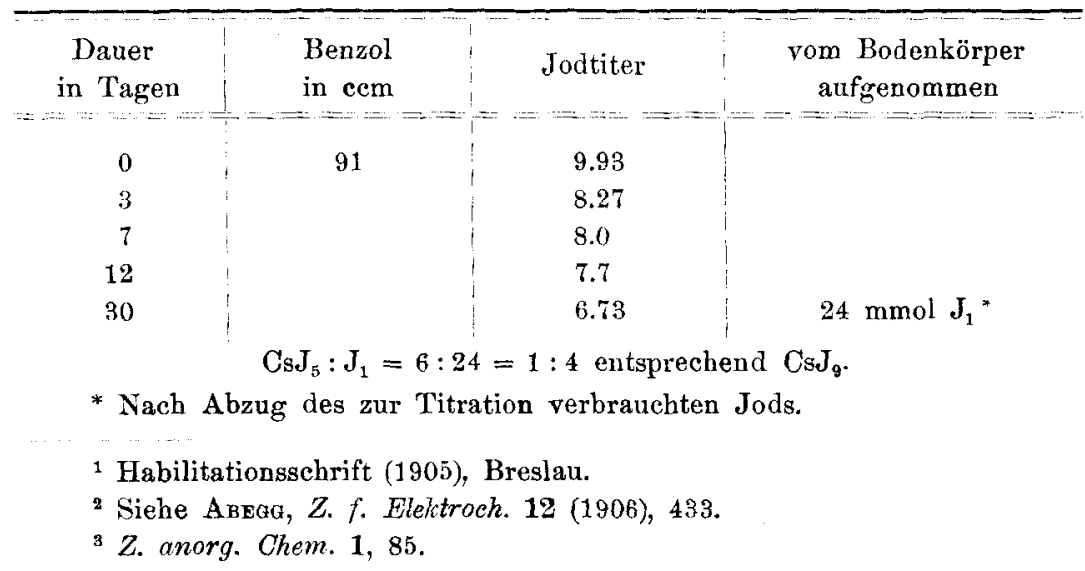


Tabelle 12.

Jodaufnahme durch $\mathrm{CsJ}_{3}$.

Am Boden $6.82 \mathrm{~g}=13.2 \mathrm{mmol} \mathrm{CsJ}_{3}$.

\begin{tabular}{|c|c|c|c|c|}
\hline $\begin{array}{c}\text { Dauer } \\
\text { in Tagen }\end{array}$ & $\begin{array}{l}\text { Benzol } \\
\text { in eem }\end{array}$ & Jodtiter & $\begin{array}{c}\text { vom Bodenkörper } \\
\text { aufgenommen }\end{array}$ & $\begin{array}{l}\text { Bodenkörper } \\
\text { Bruttoformel }\end{array}$ \\
\hline $\begin{array}{r}0 \\
60\end{array}$ & 50 & $\left.\begin{array}{r}10.32 \\
5.62\end{array}\right\}$ & 23.5 in-atom. $J_{1}$ & $\mathrm{CsJ}_{4.78}$ \\
\hline $\begin{array}{r}0 \\
12\end{array}$ & 50 & $\left.\begin{array}{r}10.78 \\
5.63\end{array}\right\}$ & 25.7 & $\mathrm{CsJ}_{6 \cdot 72}$ \\
\hline $\begin{array}{l}0 \\
8\end{array}$ & 50 & $\left.\begin{array}{r}10.53 \\
6.03\end{array}\right\}$ & 22.2 & $\mathrm{CsJ}_{8 \cdot 4}$ \\
\hline 0 & 44 & $\begin{array}{c}6.03 \\
+2.77 \mathrm{~g} \mathrm{Jod} \\
9.1\end{array}$ & 8.5 & $\mathrm{C}_{8 \mathrm{~J}} \mathrm{~J}_{9 \cdot 08}$ \\
\hline 0 & 42 & $\begin{array}{c}9.1 \\
+1 \text { g Jod } \\
10.4\end{array}$ & 2.0 & $\mathbf{C s J}_{9 \cdot 18}$ \\
\hline
\end{tabular}

$$
\begin{gathered}
81.9 \mathrm{mmol} \mathrm{J}_{1} \\
\text { auf } 13.2 \mathrm{mmol} \mathrm{Cs}_{3} \\
\mathrm{CsJ}_{3}: \mathrm{J}_{1}=13.2: 81.9=1: 6.2
\end{gathered}
$$

Tabelle 13.

\begin{tabular}{|c|c|c|c|c|c|}
\hline $\begin{array}{c}\text { Dauer } \\
\text { in Tagen }\end{array}$ & $\begin{array}{l}\text { Benzol } \\
\text { in ecm }\end{array}$ & $\begin{array}{r}\text { Jo } \\
\text { vorher }\end{array}$ & $\begin{array}{l}\text { iter } \\
\text { nachher }\end{array}$ & $\begin{array}{l}\text { vom Bodenkörper } \\
\text { abgegeb. m-atom. } J_{1}\end{array}$ & $\begin{array}{c}\text { Bruttoformel } \\
\text { d. Bodenkörpers }\end{array}$ \\
\hline 6 & 30 & 4.4 & 5.65 & 3.7 & $\mathrm{CsJ}_{8,6}$ \\
\hline 2 & 12 & 0 & 5.63 & ) & \\
\hline 2 & 15 & 0 & 5.65 & & \\
\hline 2 & 15 & 0 & 5.63 & & \\
\hline 2 & 15 & 0 & 5.62 & & \\
\hline 2 & 15 & 0 & 5.62 & 40.5 & $\mathrm{CsJ}_{4,2}$ \\
\hline 4 & 15 & 0 & 2.7 & 4.05 & $\mathrm{CsJ}_{3,8}$ \\
\hline 2 & 15 & 0 & 1.69 & & \\
\hline 14 & 10 & 0 & 1.69 & ) & \\
\hline 4 & 10 & 0 & 1.68 & 5.9 & $\mathrm{CsJ}_{8 \cdot 2}$ \\
\hline 5 & 38 & 0 & $0.62^{*}$ & 2.4 & $\mathrm{CsJ}_{2.9}$ \\
\hline
\end{tabular}

Abbau von $\mathrm{CsJ}_{9}$.

Am Boden $11.83 \mathrm{~g}=9.27 \mathrm{mmol} \mathrm{CsJ_{9 }}$.

* Dieser Titer entsprach noch nicht dem Gleichgewicht. Vermutlich war noch nicht alles $\mathrm{Cs}_{5} \mathrm{~J}_{5}$ zersetzt, aber doch auch schon CsJ entstanden. Der Versuch verunglückte, so dafs die Einstellung des $\mathrm{CsJ}_{\text {: }} \mathrm{CsJ}_{3}$-Gleichgewichts hier nicht mehr konstatiert werden konnte. 
Tabelle 14.

Abbau von $\mathrm{CsJ}_{9}$.

$4.96 \mathrm{~g} \mathrm{CsJ}_{9}$ am Boden.

\begin{tabular}{c|c|c|c|c}
\hline Dauer & Benzol & \multicolumn{2}{|c|}{ Jodtiter } & \\
in Tagen & in ccm & vorher & nachher & \\
\hline 5 & 10 & 5.1 & $\mathbf{5 . 7}$ & \\
2 & 8 & 4.4 & 5.63 & \\
6 & 10 & 4.4 & $\mathbf{5 . 6 5}$ & Mittel $\mathbf{5 . 6 5}$ \\
2 & 15 & 2.9 & $\mathbf{5 . 6 3}$ & \\
2 & 8 & 0.0 & $\mathbf{5 . 6 4}$ & \\
2 & 8 & 0.0 & $\mathbf{5 . 6 5}$ & \\
2 & 8 & 0.0 & 5.42 & \\
6 & 5 & 0.0 & 4.12 &
\end{tabular}

In dem einen Falle (Tabelle 11) war gleich von Anfang an soviel Jodlösung hinzugefügt worden, dals die Lösung, nachdem die für $\mathrm{Cs}_{9}$ nötige Menge herausgenommen war, noch über dem Gleichgewichte lag, in den beiden anderen Fällen (Tabelle 12 und 13) stellte sich bei mehreren Jodzusätzen ein Titer ein, der um weniger als $1 \%$ um den Mittelwert 5.63 schwankt. Dieser entspricht einer Lösung, die mit $\mathrm{CsJ}_{9}$ im Gleichgewicht ist, wie durch den Abbau (Tabelle 14) sichergestellt wurde; und dafs er gleichzeitig $\mathrm{CsJ}_{3}$ entspricht, geht aus Tabelle 12 mit ebenso grofser Sicherheit hervor.

Es steht hiernach fest, dals unter dem Jodtiter 0.0338 CsJ mit $\mathrm{CsJ}_{3}$ und unter $5.63 \mathrm{CsJ}_{3}$ mit $\mathrm{CsJ}_{9}$ im Gleichgewicht ist. Aufserdem findet sich beim Abbau von $\mathrm{Cs}_{9} \mathrm{~J}_{9}$ (Tabelle 13) noch ein Haltepunkt des Titers bei 1.69, der sich auch (zu 1.68) einstellte, als das auf nafsem Wege rach WeLLS und WHEkLER ${ }^{1}$ bereitete $\mathrm{Cs}_{5}$ mit Benzol längere Zeit geschüttelt wurde. Man könnte also denken, dals mit der Verbindung $\mathrm{Cs}_{5}$ diese Tension zusammenhängt, und sie sich beim Abbau von $\mathrm{CsJ}_{9}$ einstellen mülste, sobald $\mathrm{CsJ}_{9}$ aufgebraucht ist; es bliebe dann nur auffällig, dals dieses Pentajodid sich nicht regelmälsig beim Jodieren von $\mathrm{CsJ}_{3}$ bildet, welches $\mathrm{ja}$ in Tabelle 12 direkt in $\mathrm{Cs} J_{9}$ übergeht. Man könnte das durch bevorzugte Bildung von $\mathrm{Cs}_{9} J_{9}$ bei fehlenden Keimen von $\mathrm{CsJ}_{5}$ plausibel machen. Nun mülste aber nach Ostwald ${ }^{2}$ aus thermodyna-

I $Z$. anorg. Chem. 2 (1892), 255.

2 Lehrbuch d. allgem. Chemie, 2. Aufl. II, 2, S. 538. 
mischen Gründen zwischen den Tensionen der Jodierungsstufen folgende Beziehung bestehen:

$$
2 \log \mathrm{P}_{\mathrm{CsJ}_{\vartheta}, \mathrm{CsJ}_{5}}+\log \mathrm{P}_{\mathrm{CsJ}_{5}, \mathrm{CsJ}_{s}}=3 \log \mathrm{P}_{\mathrm{CsJ}}, \mathrm{Css}_{8} .
$$

Setzt man hierin

$$
\mathrm{P}_{\mathrm{Cs}_{9}, \mathrm{Cs}_{3}}=5.63, \mathrm{P}_{\mathrm{Cs}_{5}, \mathrm{CsJ}_{3}}=1.69
$$

so berechnet sich

$$
\mathrm{P}_{\mathrm{Cs}_{\mathbf{9}}, \mathrm{Cs}_{\mathrm{j}}}=10.3 \text {. }
$$

Dieser Wert scheint also angesichts der Tabelle 11 aus. geschlossen; denn hier wird $\mathrm{CsJ}_{5}$ unter erheblich kleineren Jod-

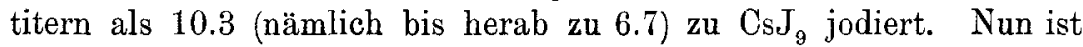
aber nach den Erfahrungen beim Rb-Polyjodid möglich, dals das Präparat nach Wells und WreELER noch gar kein Individuum war, d. h. sich noch im Dissoziationszustand in Gestalt einer Mischung von $\mathrm{CsJ}_{3}+\mathrm{J}_{2}$ befand. Dafür spricht schon die Angabe dieser Autoren, dafs dieses Präparat Joddampf ebenso schnell wie reines Jod abgibt, während es nach dem Gleichgewichtstiter 1.69 nur etwa 0.154 der Tension des reinen Jods haben könnte. Mit dieser Deutung sind alle unsere Resultate im Einklang.

Die Existenz einer Jodierungsstufe zwischen $\mathrm{CsJ}_{3}$ und $\mathrm{CsJ}_{9}$ wurde auch erwiesen, indem $\mathrm{CsJ}_{3}$ mit Jod-Benzollösung rom ungefähren Titer 6 geschüttelt auf den konstanten Titer 1.71 herabging. Alle diese $\nabla$ ersuche erweisen allerdings nicht, ob die Zwischenstufe $\mathrm{Cs}_{5}$ oder vielleicht $\mathrm{Cs}_{7}$ ist. Infolge der grofsen Trägheit, mit der sich diese Zwischenstufe bildet, wurde die Untersuchung in diesem Punkte nicht zu Ende geführt.

Es existieren also bei $25^{\circ}$ folgende festen Alkali-Polyjodide:

\begin{tabular}{l|c|c|c|c}
\hline & $J_{3}$ & $J_{5}$ & $J_{7}$ & $J_{9}$ \\
\hline \hline $\mathrm{Li}$ & - & - & - & - \\
$\mathrm{Na}$ & - & - & - & - \\
$\mathrm{K}$ & - & - & $\mathrm{KJ}_{7}$ & - \\
$\mathrm{NH}$ & $\mathrm{NH}_{4} \mathrm{~J}_{3}$ & - & - & \\
$\mathrm{Rb}$ & $\mathrm{RbJ}_{3}$ & - & $\mathrm{RbJ}_{7}$ & $\mathrm{RbJ}_{9}$ \\
$\mathrm{Cs}$ & $\mathrm{CsJ}_{3}$ & $\left(\mathrm{CsJ}_{5}\right.$ oder $\left.\mathrm{CsJ}_{7}\right)$ & $\mathrm{CsJ}_{9}$
\end{tabular}

Die oben (meist in $0.1 \mathrm{n}$. Thiosulfattiter) gefundenen Werte liefern nach Umrechnung in relative Jodtensionen folgende Zusammenstellung: 


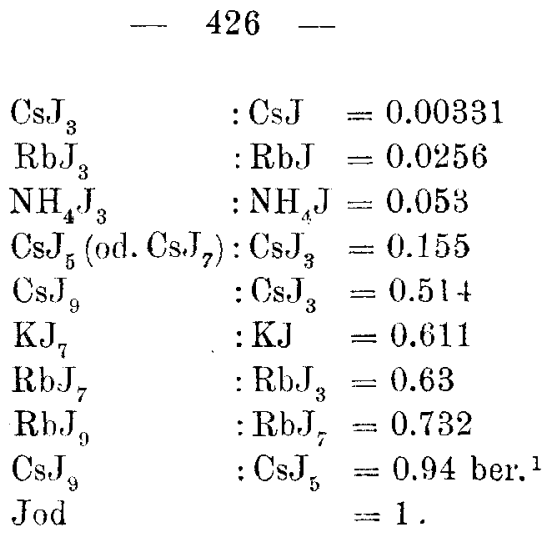

Die hier angewandte Methode empfiehlt sich besonders zur Aufsuchung solcher Verbindungen, die durch hohe Temperaturen soweit zerfallen, dafs sie sich bei der thermischen Analyse (siebe Teil III) nicht mehr auffinden lassen. Sie würde sich z. B. auch für die Untersuchung der Polysulfide, die soeben auf andere wohl weniger bequeme Weise von W. BILTz und WiLkE-Dörfurt ${ }^{2}$ ausgeführt ist, oder der Polyborate ${ }^{3}$ eignen.

\section{Konzentrierte Lösungen von Jod und Jodkalium in Wasser.}

Die bei der Untersuchung der Kalium-Polyjodide auftretenden Störungen infolge des Eindringens von Wasser in die Flaschen veranlafsten uns, die Löslichkeit von Jod in sehr konzentrierten Jodkaliumlösungen und die von $\mathrm{KJ}$ in starken Jodlösungen zu untersuchen, um das Optimum der Polyjodidbildung in der Lösung, d. h. den grölsten Wert des Verhältnisses $J: K J$ festzustellen und die Konzentration an Jod und Jodkalium, die diesem Verhältnis entspricht.

Bei den grofsen Mengen von KJ und Jod, die bei den Benzolversuchen immer angewendet wurden, konnten geringe Wassermengen, wie sie durch den Stopfen in die Flaschen eindringen, nur gesättigte Lösungen bilden.

Schon Dossios und Weite ${ }^{4}$ haben im Jahre 1869 festgestellt, dals die Löslichkeit von $J$ in Jodkaliumlösungen mit zunehmenden KJ-Gehalt stark zunimmt. Für verdünnte KJ-Lösungen von 0.1

1 Für $\mathrm{CsJ}_{9}: \mathrm{CsJ}_{7}$ würde sich ergeben $=5.6$ also viel über Joddruck!

2 . anorg. Chem. 48 (1906), 297.

${ }^{3}$ Auerbach, Z. anorg. Chem. 37, 353; P. Mueller, Dissert. Breslau.

4 Dammer, Handbuch. 
bis $0.005 \mathrm{n}$ haben dann Noyes und SeidpenstickeR ${ }^{1}$ gefunden, dafs das Verhältnis $J: K J$ konstant und kleiner als $1 \mathrm{~J}_{2}: 1 \mathrm{KJ}$ ist, was mit den Resultaten von $J_{A K O W K I N}{ }^{2}$ übereinstimmt, der für dieses Konzentrationsbereich eine gut stimmende Dissoziationskonstante für $\mathrm{KJ}_{3} \rightleftarrows \mathrm{KJ}+\mathrm{J}_{2}$ berechnen konnte.

BRUNER $^{3}$ hat die Angaben von Dossios und Weith bestätigt und festgestellt, dafs das Verhältnis $\mathrm{J}: \mathrm{KJ}$ eine Funktion der KJKonzentration ist und zwar gleichsinnig mit dieser wächst.

Seine Zahlen, sowie die von Dossros und $W_{\text {EITH }}$ beziehen sich auf 0.06 bis $0.745 \mathrm{n}$. KJ-Lösungen; wir haben KJ-Lösungen von 1.9 bis 5.5 n. untersucht und solche, die KJ als Bodenkörper hatten und wechselnde Mengen Jod enthielten. Die Tabelle 15 zeigt die erhaltenen Werte, die ersten sind von Bruner, WeITH und Dossios, aber auf Normalität umgerechnet, um ihre Werte, die teils in Prozentgehalt in Gramm, teils in Normalität angegeben waren, mit den unsrigen vergleichbar zu machen:

Tabelle 15.

Löslichkeit von Jod in KJ-Lösungen.

\begin{tabular}{|c|c|c|c|c|}
\hline $\mathrm{Nr}$ & n. $\mathbf{K J}$ & n. J-A tome & KJ : J & \\
\hline 1 & 0.06 & $0.06^{2}$ & $1: 1$ & \multirow{6}{*}{ Bruner } \\
\hline 2 & 0.12 & 0.125 & $1: 1.04$ & \\
\hline 3 & 0.24 & 0.25 & $1: 1.04$ & \\
\hline 4 & 0.36 & 0.39 & $1: 1.08$ & \\
\hline 5 & 0.48 & 0.545 & $1: 1.08$ & \\
\hline 6 & 0.60 & 0.7 & $1: 1.16$ & \\
\hline 7 & 0.665 & 0.78 & $1: 1.17$ & \multirow{3}{*}{$\begin{array}{c}\text { Dossos u. } \\
\text { WEITH }\end{array}$} \\
\hline 8 & 0.718 & 0.88 & $1: 1.23$ & \\
\hline 9 & 0.765 & 0.95 & $1: 1.24$ & \\
\hline 10 & 1.91 & 3.29 & $1: 1.72$ & \multirow{5}{*}{ HAMBURGER } \\
\hline 11 & 2.85 & 5.45 & $1: 1.92$ & \\
\hline 12 & 4.51 & 11.52 & $1: 2.55$ & \\
\hline 13 & 5.36 & 17.12 & $1: 3.19$ & \\
\hline 14 & 5.55 & 17.16 & $1: 3.1$ & \\
\hline
\end{tabular}

Die Löslichkeitsbestimmungen wurden so ausgeführt, dals Jodkaliumlösungen verschiedener Konzentrationen mit Jod gesättigt und ihr Jodgehalt titriert wurden. $\mathrm{Zu}$ diesen wurde dann, um Punlte

1 1. c.

${ }^{2}$ l. c.

${ }^{3}$ Zeitschr. phys. Chem. 26, 150. 
Tabelle 16.

Löslichkeit von $\mathrm{KJ}$ in Jodlösungen.

\begin{tabular}{c|c|c}
\hline n. KJ & n. J-Atome & KJ : J \\
\hline \hline 6.15 & 0.00 & \\
6.23 & 3.64 & $1: 0.585$ \\
6.40 & 11.11 & $1: 1.735$ \\
6.36 & 13.16 & $1: 2.07$ \\
6.33 & 13.2 & $1: 2.08$ \\
6.24 & 17.03 & $1: 2.73$
\end{tabular}

der KJ-Sättigungskurve zu erhalten, KJ im Überschufs hinzugefügt. Die Lösungen waren tief dunkelblau und zeigten perlmutterartigen Schimmer; sie waren vollkommen undurchsichtig, so dafs mit dem Auge nie festgestellt werden konnte, ob Bodenkörper vorhanden war. Die spezifische Gewichtsbestimmung der Lösung 1:3.10 (Tabelle 15, Nr. 14) ergab den aufserordentlich hohen

Gegenseitige Löslichkeitsbeeinflussung von Jod und Jodkalium in Wasser.

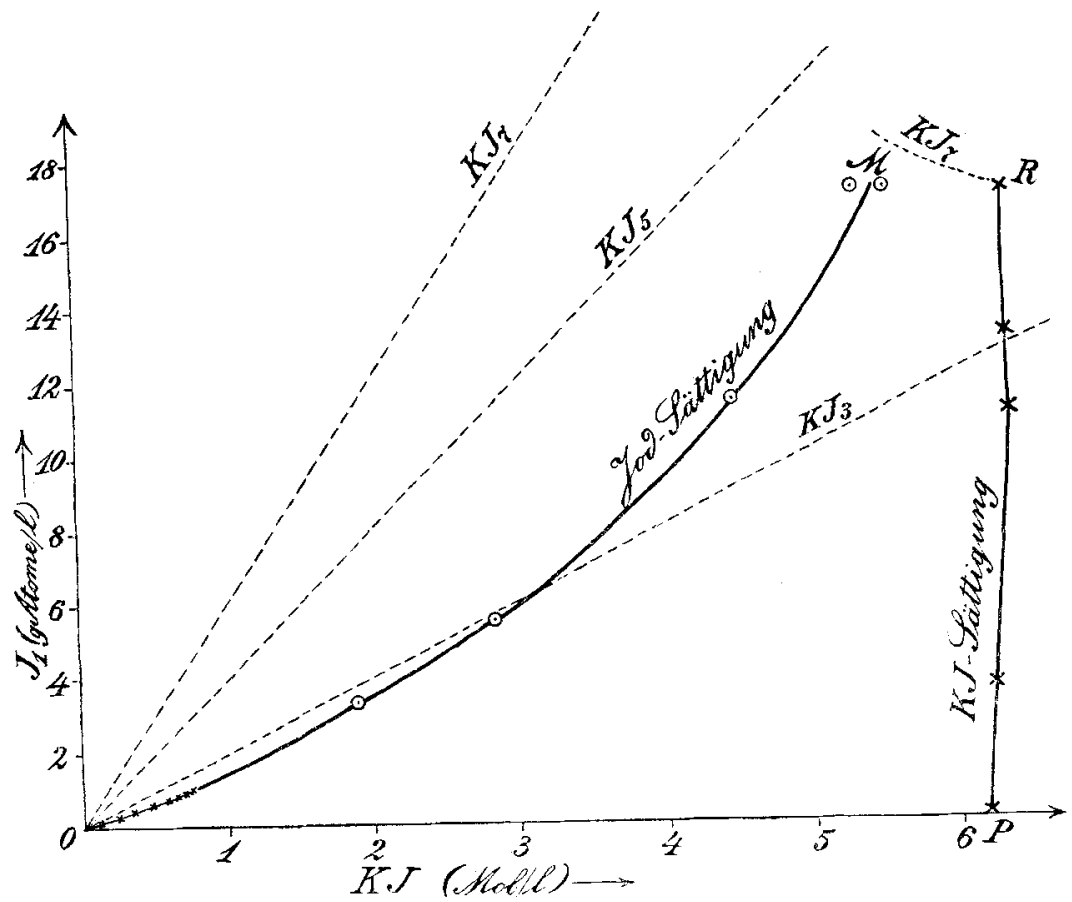

Fig. 2. 
Wert von 2.75. Zur Titration wurde $1 \mathrm{ccm}$ mittels einer für Aufnahme geeichten Pipette in einen $10 \mathrm{ccm}$-Kolben gebracht und nach Hinzufügen einer abgewogenen Menge Jodkalium, um das Ausfallen von Jod zu verhindern, mit Wasser aufgefüllt. Dazu ist bei an Jod sehr konzentrierten Lösungen, wie aus der Kurve ersichtlich, oft viel mehr KJ nötig, als die Lösung enthält. So z. B. im extremen Falle 13 war durch Verdünnen auf das 10 fache Volumen die Lösung $1.71 \mathrm{n}$. an Jod und $0.54 \mathrm{n}$. an KJ. Eine $1.71 \mathrm{n}$. Jodlösung ist aber nur in einer an Jodkalium ca. $1.5 \mathrm{n}$. Lösung möglich. Es mufste also mindestens doppelt so viel Jodkalium hinzugefügt werden, als die Lösung vorher enthielt, um das Ausfallen von Jod zu verhindern.

Titriert wurde dann in $1 \mathrm{ccm}$ dieser Lösung zuerst das Jod mit Thiosulfat und dann das gesamte Jodion mit Silbernitrat. Es wurden immer mehrere Titrationen von jeder Lösung gemacht und gute Übereinstimmung gefunden. Der Jodgehalt war direkt aus dem verbrauchten $\mathrm{Na}_{2} \mathrm{~S}_{2} \mathrm{O}_{3}$ berechenbar, also recht genau bestimmbar. Die KJ-Konzentration berechnete sich aus der Anzahl Mol verbrauchten $\mathrm{AgNO}_{3}$ (Mol. verbr. Thios. + Mol zugefügten $\mathrm{KJ}$ ).

Die Versuchsfehler liegen hier einerseits in der Pipettierung von so ungeheuer konzentrierten Lösungen, wo schon dadurch, dafs man nicht nur bis zur Marke aufsaugt, sondern etwas höher und dann abfliefsen läfst, die Benetzung uber die Marke einen merklichen Fehler bedingt. Andererseits liegen sie für die Jodkaliumbestimmungen in dem $\mathrm{Um}$ stande, dafs sie als Differenz der zwei grofsen Zahlen: GesamtJodionkonz. - (Jodkonz. + hinzugewogenes Jodkalium) bestimmt wird. Anwendung von geeigneten Pipetten und Bestimmung von $J$ und $\mathrm{KJ}$ in getrennten Portionen hätten diese Fehler stark reduziert; da es für unseren Zweck aber nur auf einen Überblick über die Verhältnisse ankam, so begnügten wir uns mit den bei sorgsamem Arbeiten auf obige Weise erhaltenen Resultaten.

Tabelle 16 enthält die Löslichkeit von Jodkalium in verschieden konzentrierten Jodlösungen bei $25^{\circ}$.

Zunächst bestimmten wir die Löslichkeit von $\mathrm{KJ}$ in Wasser; die vorhandenen Bestimmungen von MULDER und von DE COPPET ${ }^{1}$, die Gramm $\mathrm{KJ}$ in $100 \mathrm{~g}$ Wasser angeben, waren nicht verwendbar, da wir die Normalität einer gesättigten Lösung kennen mulsten. Aus alten spezifischen Gewichtsbestimmungen starker Jodkaliumlösungen

${ }^{1}$ Landoldt Börnstein, III. Aufl, S. 545. 
von Kremers ${ }^{1}$ und den Zahlen von MoLder berechnete sich eine mit der von uns gefundenen gut übereinstimmende Löslichkeit.

Eine bei $25^{\circ}$ gesättigte KJ.Lösung ist danach $6.15 \mathrm{n}$. an KJ, enthält also $1002 \mathrm{~g}$ im Liter Lösung.

Tragen wir die Werte der Tabellen in ein Koordinatensystem ein (Kurve S. 428), dessen Abzissen den KJ-Gehalt und dessen Ordinaten den Jodgehalt der Lösungen bedeuten, so stellt die Kurve $O M$ die Löslichkeitskurve von Jod bei steigendem Zusatz von Jodkalium und $P R$ die Löslichkeitskurve von Jodkalium bei steigendem Zusatz von Jod dar. Längs $O M$ ist Jod Bodenkörper, längs $P R$ Jodkalium. Von der Kurve $M R$ ist nur der Punkt $R$ bestimmt, der übrige Verlauf hypothetisch.

Die einzige oben von uns festgestellte Verbindung von Jod und $\mathrm{KJ}$ ist $\mathrm{KJ}_{7}$. Da das Zusammensetzungsverhältnis der Lösung den diesem Bodenkörper entsprechenden Wert $\mathrm{KJ}: \mathrm{J}_{1}=1: 6$ in der Nähe der gemeinsamen Sättigung nicht entfernt erreicht, so befindet sich bei $25^{\circ} \mathrm{KJ}_{7}$ - als Doppelsalz aufgefalst - in seinem Umwandlungsintervall, es ist ohne, und zwar grolsen, Überschufs einer seiner Komponenten in Berührung mit Wasser nicht beständig, sondern spaltet Jod ab.

Wie die Tabellen und die Kurve zeigen, liegt die Zusammensetzung der jodgesättigten Lösungen bis über $3 \mathrm{n}$. KJ unterhalb der Formel $\mathrm{KJ}_{3}$ und bleibt selbst bis in die Nähe der KJ-Sättigung noch weit unter $\mathrm{KJ}_{7}$. Ganz im Anfang schneidet die Jodkurve natürlich einmal sämtliche punktiert eingezeichneten Linien für die Verhältnisse $\mathrm{KJ}_{3}$, wie $\mathrm{KJ}_{5}$ und $\mathrm{KJ}_{7}$, da für $\mathrm{KJ}=0$ die Löslichkeit $\mathbf{J}$ einen endlichen wenn auch sehr kleinen Wert, nach Noyes und Serdenstrcker ${ }^{3}$ 0.00134 n., besitzt.

Auffallend vom molekulartheoretischen Standpunkt ist die verschwindend kleine Löslichkeitsbeeinflussung des KJ durch Jod. Man ist versucht zu denken, dals die Konstanz des Löslichkeitsproduktes $(\mathrm{K}) \cdot\left(\mathrm{J}^{\prime}\right)$ in der KJ-gesättigten Lösung eine starke Erhöhung der $\mathrm{K}$-Konzentration verlangen sollte, wenn durch Jodzusatz $\mathrm{J}^{\prime}$-Ionen in Polyjodid-Ionen verwandelt werden. Zur Erklärung müfste man annehmen, dafs $\mathrm{KJ}_{3}$ resp. die anderen in der Lösung nebeneinander sich bildenden Polyjodide viel stärker als $\mathrm{KJ}$ ionisiert wären, oder dafs die Änderung des Lösungsmittels durch die Zusätze ent-

1 Pogg. Ann. 108 (1859), 115.

${ }^{2}$ Zeitschr. phys. Chem. 27, 359 . 
sprechend löslichkeitsvermindernd einwirkt. Die erstere an sich nicht unplausible Deutung stebt aber im Widerspruch mit Befunden von $D_{A w s o n}{ }^{1}$, der für die Polyjodide geringere Ionisation als für Monojodid fand. Für den letzten auf der KJ-Kurve bestimmten Punkt wurde durch Schütteln mit Benzol die Jodtension gleich dem Gleichgewicht $\mathrm{KJ}_{2}: \mathrm{KJ}$ festgestellt. Es ist also hier aufser $\mathrm{KJ}$ noch $\mathrm{KJ}_{7}$ Bodenkörper, d. h. hier schneidet die Löslichkeitskurve von $\mathrm{KJ}_{7}$ diejenige von $\mathrm{KJ}$. $\mathrm{W}_{0}$ die $\mathrm{KJ}_{7}$-Kurve die $J$-Kurve schneidet, ist nicht festgestellt worden, da dies von unserem Ziel zu weit abgeführt hätte. Doch erscheint es lohnend, die phasentheoretische Untersuchung des Systems $\mathrm{KJ}+J$ in dieser Richtung und für verschiedene Temperaturen noch weiter zu verfolgen. Wenn überhaupt bei erreichbarer Temperatur, so dürfte erst erheblich unterhalb $25^{\circ}$ $\mathrm{KJ}_{7}$ aus dem Umwandlungsintervall in das Gebiet der Stabilität gelangen.

\section{Erstarrungskurven von Jod-Jodkaliumgemischen.}

Die Aufnahme der Schmelz- und Erstarrungskurven von wechselnden Mengen der Bestandteile ist eine weitere Methode ${ }^{2}$ zur Entdeckung fester Verbindungen, die besonders von Roozeboom, Tamman und ihren Mitarbeitern mit Erfolg benutzt wurde.

Wir benutzten auch diese Methode für einige Fälle in folgender Weise :

Abgewogene Mengen von Jod und Jodkalium wurden in einem in der Mitte etwas verengten Rohr innig gemischt, und ein mit Asbestwolle umwickeltes Thermometer so hineingesteckt, dafs der Asbestpfropfen auf der Verengung der Röhre aufsals, um das Verdampfen von Jod zu hindern. Die beim Erhitzen in den Asbestpfropfen destillierende Jodmenge war im Verhältnis zu seiner Ge. samtmenge zu vernachlässigen, da immer 20-30 g Substanz angewendet wurden. Die Erhitzung geschah in einem grofsen Sandbade, das gut mit Asbestpapier umwickelt war, um die Abkühlung möglichst zu verlangsamen. Die Röhre mit der Schmelze wurde mittels eines Korkstopfens in ein weiteres Rohr eingesetzt, um sie mit einem Luftmantel zu umgeben, und dieses bis zum Rande in

1 Centrbl. 1991 I, 214.

2 Kremann, der sie ebenfalls mehrfach benutzte und Ber. deutsch. chem. Ges. 39 (1906), 1023 meint, dafs es die einzige sei, hat die auch sehr allgemeine oben angewandte Tensionsmethode jedenfalls übersehen. 
das Sandband vergraben und mit Asbestpappe zugedeckt. Die Temperatur wurde mit einem einfachen, in ganze Grade geteilten Thermometer gemessen, bei dem Ablesen mit der Lupe $0.1^{\circ}$ noch gut zu schätzen erlaubte. Die Abkühlung im Sandbade wurde messend verfolgt. Die Schmelze wurde während des Erstarrens mit dem Thermometer oder einem Rührer aus starkem Platindraht, der um das Thermometer gewickelt war, gerührt; trotzdem traten immer mehr oder weniger starke Unterkühlungen ein. Die Temperatur wurde jede halbe oder ganze Minute notiert und jede Kurve dreimal aufgenommen, wobei recht gut übereinstimmende Werte für die Knickpunkte erhalten wurden. Die Dauer der Erstarrung des Eutektikums betrug oft bis $1 / 2$ Stunde, während vor- und nachher die Temperatur pro Minute um $1 / 2^{0}$ gesunken war. Im folgenden sind die Zahlen von zwei Versuchen angeführt:

1. Gehalt: $22.3 \mathrm{Mol} \% \mathrm{KJ}$.

\begin{tabular}{|c|c|c|c|c|c|c|c|}
\hline $\begin{array}{c}\text { Zeit } \\
\text { in Min. }\end{array}$ & $\begin{array}{l}\text { Temp. } \\
\text { in }{ }^{0}\end{array}$ & $\begin{array}{c}\text { Zeit } \\
\text { in Min. }\end{array}$ & $\begin{array}{c}\operatorname{Temp} \\
\operatorname{in}{ }^{0}\end{array}$ & $\begin{array}{c}\text { Zeit } \\
\text { in Min. }\end{array}$ & $\begin{array}{c}\text { Teinp. } \\
\text { in }{ }^{0}\end{array}$ & $\begin{array}{c}\text { Zeit } \\
\text { in Min. }\end{array}$ & $\begin{array}{l}\text { Temp. } \\
\text { in }{ }^{\circ}\end{array}$ \\
\hline 0 & 94 & 11 & 83.5 & 22 & 81.3 & 33 & 81 \\
\hline 1 & 93.2 & 12 & 83 & 23 & 81.3 & 34 & 80.7 \\
\hline 2 & 92.2 & 13 & 82.1 & 24 & 81.3 & 35 & $80 . \tilde{5}$ \\
\hline 3 & 91 & 14 & 81.5 & 25 & 81.3 & 36 & 80.2 \\
\hline 4 & 90.2 & 15 & 81 & 26 & 81.3 & 37 & 80 \\
\hline 5 & 89.2 & 16 & 80.9 & 27 & 81.3 & 38 & 79.7 \\
\hline 6 & 88.2 & 17 & 81.1 & 28 & 81.3 & 40 & 78.7 \\
\hline 7 & 87.2 & 18 & 81.3 & 29 & 81.3 & 42 & 77.5 \\
\hline 8 & 86.2 & 19 & 81.5 & 30 & 81.3 & 44 & 75 \\
\hline 9 & 85.2 & 20 & 81.3 & 31 & 81.3 & 46 & 72 \\
\hline 10 & 84.2 & 21 & 81.3 & 32 & 81.3 & 47 & 69.5 \\
\hline
\end{tabular}

2. Gehalt: $11.1 \mathrm{Mol} \% \mathrm{KJ}$.

\begin{tabular}{|c|c|c|c|c|c|c|c|}
\hline 0 & 113 & 15 & 99.5 & 30 & 95.2 & 45 & 81.5 \\
\hline 1 & 112 & 16 & 99.7 & 31 & 94.7 & 46 & 81 \\
\hline 2 & 111 & 17 & 100.2 & 32 & 94 & 47 & 80.5 \\
\hline 3 & 110 & 18 & 100.2 & 33 & 93.5 & 48 & 80.2 \\
\hline 4 & 109.2 & 19 & 100 & 34 & 92.7 & 49 & 80.2 \\
\hline$\check{\mathbf{s}}$ & 108.2 & 20 & 99.7 & 35 & 91 & 50 & 80.5 \\
\hline 6 & 107.3 & 21 & 99.5 & 36 & 90.5 & 51 & 80.6 \\
\hline 7 & 106.2 & 22 & 99.2 & 37 & 89.2 & 52 & 80.7 \\
\hline 8 & 105.5 & 23 & 99 & 38 & 88.5 & 53 & 80.5 \\
\hline 9 & 104.7 & 24 & 98.5 & 39 & 87.5 & 54 & 80 \\
\hline 10 & 103.9 & 25 & 98 & 40 & 86.9 & 50 ว & 79.7 \\
\hline 11 & 102.9 & 26 & 97.5 & 41 & 85.9 & 56 & 78.5 \\
\hline 12 & 101.2 & 27 & 97 & 42 & 84 & 57 & 77.9 \\
\hline 13 & 101.2 & 28 & $96 . \overline{5}$ & 43 & 83.2 & 58 & 76.5 \\
\hline 14 & 100.2 & 29 & 96 & 44 & 82. & 59 & 75 \\
\hline
\end{tabular}

Die Zahlen zeigen in Versuch 1 einen scharfen Haltepunkt bei $81.3^{\circ}$, der auch bei zwei weiteren Versuchen mit derselben Schärfe 
ausgeprägt war. Versuch 2 zeigt zwei Haltepunkte bei $100.2^{\circ}$ und ca. $80.5^{\circ}$, die gleichfalls in mehrmaligen Bestimmungen bis auf wenige Zehntel Grad wiederkehrten. Das aus sämtlichen Versuchen zusammengestellte Schmelzdiagramm (Kurve 3) zeigt, dals zuerst, entsprechend der Theorie, eine Schmelzpunkterniedrigung des Jods auftritt. Die in diesem Intervall aufgenommenen Kurven, zu denen auch die von Versuch 2 gehört, zeigen die im Diagramm verzeichneten zwei Knickpunkte beim Anfang- und Endpunkt der Erstarrung.

Schmelzpunkte von Jod-Jodkaliumgemischen.

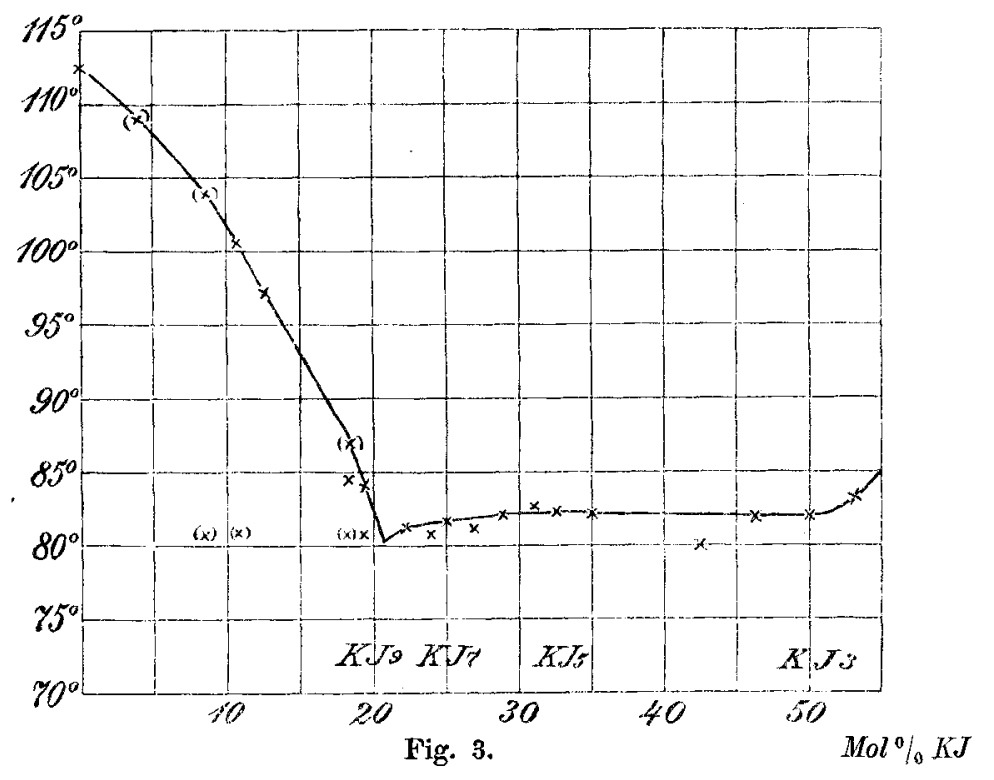

Das Eutektikum liegt bei ca. $80.5^{\circ}$. Die Zusammensetzung der Schmelze, die dem Eutektikum entspricht, ist etwas jodärmer als $\mathrm{KJ}_{9}$, was auf die Nichtexistenz dieser Verbindung hindeutet. Wäre überhaupt keine Verbindung zwischen KJ und $J$ vorhanden, so mülste jetzt der aufsteigende Ast der Schmelzkurve von KJ beginnen; dafür sind zwar keine Anzeichen vorhanden, es zeigt sich aber auch kein ausgeprägtes Maximum, sondern alle Schmelzen mit steigendem Jodkaliumgehalt erstarren bei konstanter Temperatur, die von dem früher gefundenen Eutektikum bei $80.5^{\circ}$ nach und nach etwas ansteigt, bis sie kurz hinter $\mathrm{KJ}_{7}$ entsprechenden Gemischen deutlich etwas über $82^{\circ}$ liegt. Nur bei der letzten untersuchten Schmelze mit dem gröfsten Jodkaliumgehalt wurde $83^{\circ}$ gefunden. 
In der folgenden Tabelle und in der Kurventafel Fig. 3 konsten wir aufser unseren $(\mathrm{H})$ auch eine Reihe von Bestimmungen $(\mathrm{K})$ aufnehmen, die Hr. J. KopPEL-Berlin unabhängig von uns gemacht hatte, ehe er erfuhr, dals wir damit bereits beschäftigt waren. Wie man sieht, stehen sie mit den unseren in fast absoluter Übereinstimmung. Die Kurvenpunkte, die von uns beiden bestimmt wurden, sind in ( ) gestellt.

\begin{tabular}{|c|c|c|c|c|}
\hline & $\begin{array}{c}\text { KJ } \\
\text { Mol.- } \%\end{array}$ & $\begin{array}{l}\text { Gef. } \\
\%\end{array}$ & $\begin{array}{l}\text { Schmelzp. } \\
\text { (Anfangsp.) }\end{array}$ & $\begin{array}{l}\text { Haltep. } \\
\text { (Eutekt.) }\end{array}$ \\
\hline $\mathrm{K}$. & 0 & 0 & $112^{\circ}$ & - \\
\hline H. & 0 & 0 & 112.5 & - \\
\hline $\mathbf{K}$. & 3.9 & 2.6 & 109.1 & - \\
\hline $\mathrm{K}$. & 8.5 & 5.7 & 104 & 80.9 \\
\hline K. & 10.8 & 7.4 & 100.6 & 80.6 \\
\hline H. V & 11.1 & 7.5 & 100 & 80.7 \\
\hline H. I & 12.6 & 8.6 & 97 & nicht beob. \\
\hline H. VI & 18.5 & 12.9 & 84.5 & 80.8 \\
\hline K. & 18.5 & 12.9 & 87 & 80.7 \\
\hline H. IX & 19.4 & 13.6 & 84 & 80.8 \\
\hline H. VII & 22.3 & & & 81.3 \\
\hline H. III & 24.2 & & sehr starke Unterkühlung & {$[80.5]$} \\
\hline H. XVI & 25.0 & & & 81.3 \\
\hline H. II & 27.3 & & sehr geringe Mengen & {$[81]$} \\
\hline H. VIII & 28.4 & & & 82 \\
\hline H. XVII & 31.0 & & & 82.5 \\
\hline H. XIII & 32.4 & & & 82.2 \\
\hline H. XV & 35 & & & 82 \\
\hline H. XI & 42.6 & & & $(80)$ \\
\hline H. IV & 45.7 & & & 81.9 \\
\hline H. XII & 50.0 & & & 82 \\
\hline H. XIV & 53.0 & & & 83.2 \\
\hline
\end{tabular}

Weitere Schmelzen wurden bisher nicht untersucht, weil, wie Versuche im zugeschmolzenen Rohr gezeigt hatten, diese erst bei viel höheren Temperaturen schmelzen, so dafs im offenen Rohr das Jod wegdampfen würde. Es sind also Anzeichen dafür vorhanden, dals hier die Jodkaliumschmelzkurve anzusteigen beginnt.

KnEManN ${ }^{1}$ hat nun in ausgedehnten Untersuchungen über die Schmelzen dissoziierender Verbindungen festgestellt, dafs ihre Schmelzkurven ein mehr oder weniger abgeflachtes Maximum zeigen, das dadurch erklärlich ist, dafs die Dissoziationsprodukte Schmelzpunkt erniedrigend wirken. Er hat Fälle beobachtet, wo die Schmelzkurve der chemischen Verbindung durch die gerade Ver- 
bindungslinie der beiden Eutektika dargestellt wird; auch findet er oft um weniger als $1^{0}$ voneinander entfernt liegende Eutektika. So liegen beim Gemisch Trinitrobenzol + Naphthalin die Eutektika bei 71 und $72^{\circ}$, beim m. Dinitroluol + Naphthalin liegt das eine Eutektikum bei $50.3^{\circ}$, das andere bei $50.5^{\circ}$ und das Maximum bei $50.8^{\circ}$.

Analog dürfen wir annehmen, dafs in unserem Falle $80.5^{\circ}$ und $82^{\circ}$ die Eutektika sind and das Maximum infolge der starken Dissoziation der Schmelze so sehr erniedrigt ist, dafs der Beginn der Erstarrung nur wenig über dem eutektischen Punkt liegt und deshalb nicht deutlich als zweiter Knickpunkt der Erstarrungskurven wahrnehmbar wird. Über das Vorhandensein eines bestimmten Polyjodides in der Schmelze geben deshalb die Kurven keinen genauen Aufschlufs. Sie stehen jedoeh mit den Resultaten der Tensionsbestimmungen im besten Einklang, insofern $\mathrm{KJ}_{7}$ zwischen den beiden Eutektika liegt und die Schmelze, deren Kurventabelle durch Versuch 1 gegeben ist und die nahezu der Zusammensetzung $\mathrm{KJ}_{7}$ entspricht, einen ganz besonders gut ausgeprägten Erstarrungspunkt bei $81.3^{\circ}$ zeigt. Es liegen also alle Anzeichen für das Vorhandensein von $\mathrm{KJ}_{7}$ auch hier vor, um so mehr, wenn man bedenkt, wie stark diese schon bei $25^{\circ}$ dissoziierende Verbindung bei ca. $80^{\circ}$ gespalten sein muls.

Jedenfalls ist aus den Kurven ersichtlich, dafs Jod und KJGemische nicht unterhalb $80^{\circ}$ schmelzen können.

Damit sind die Schmelzpunktsbestimmungen von JoHNson ${ }^{1}$ und $W_{\text {ELLSS }}{ }^{2}$ für ein $\mathrm{KJ}_{3}$, die dessen Schmelzpunkt bei 45 resp. $38^{\circ}$ angeben, als falsch erkannt. Sie sind zweifellos durch Wassergehalt entstellt; wir beobachteten sogar bei reinem aus Wasser auskristallisierenden Präparat schon bei ca. $30^{\circ}$ Schmelzung in der Kapillare.

In der Tat kann man durch blofses Behauchen eines Jodkalium-Jodgemisches seinen Schmelzpunkt von 80 auf $40^{\circ}$ und tiefer herunterdrücken.

Die Erstarrungskurven von $\mathrm{RbJ}_{9}$ zeigen einen Schmelzpunkt von $81.3^{\circ}$, der sich bei zwei Versuchen sehr scharf einstellte. Erstarrungskurven von Cäsiumpolyjodiden wurden nicht aufgenommen, jedoch weist die Angabe von WELLs, dafs alle künstlichen Gemische von der Zasammensetzung $\mathrm{CsJ}_{4}$ bis $\mathrm{CsJ}_{9}$ durchweg bei der gleichen Temperatur von $73^{\circ}$ schmelzen, darauf hin, dals dort die Verhältnisse ähnlich liegen, wie beim Kalium. Der Ansicht

1 Journ. Chem. Soc. 1877 I, 249.

"Z. anorg. Chem. 1, 442. 
von WELLS ${ }^{1}$, es sei höchst merkwürdig, dafs dieser Schmelzpunkt bei $73^{\circ}$ liege, also niedriger als der von $\mathrm{CsJ}_{3}$ und Jod, können wir heute natürlich nicht mehr beistimmen, da wir wissen, dals ein Eutektikum immer niedriger schmelzen mufs als seine Bestandteile.

Von Interesse ist auch, die Schmelzpunktserniedrigung des Jods durch den Jodidzusatz mit der nach vas'x HowF berechenbaren zu vergleichen. Die Molarerniedrigung $E$ des Jods beträgt, da sein Schmelzpunkt in absoluter Temperaturzählung $386^{\circ}$, seine Schmelzwärme $11.7 \mathrm{cal} . / \mathrm{g}$ beträgt

$$
E=\frac{2 \cdot 386^{2}}{1000 \cdot 11.7}=25.4^{0}
$$

für 1 Mol gelösten Stoffes in $1000 \mathrm{~g}=\frac{1000}{254}=4 \mathrm{Mol} \mathrm{J}{ }_{2}$. Es ergeben sich daraus für die Schmelzpunktserniedrigungen

$\begin{array}{crrrrr}\Delta= & 3^{\circ} & 8^{0} & 12^{\circ} & 25^{\circ} & 32^{\circ} \\ \text { berechnet für. KJ \% } \% 2.9 & 7.4 & 10.7 & 20 & 24 \\ \text { statt der angew. KJ \% } \% 3.9 & 8.5 & 10.8 & 18 & 21\end{array}$

also in ziemlich naher Übereinstimmung. Bei geringen KJ-Konzentrationen wäre danach die Depression zu klein, bei grofsen zu hoch. Ersteres könnte auf Assoziation der KJ-Molekeln deuten, letzteres von der Bindung der lösenden $\mathrm{J}_{2}$-Molekeln an das gelöste $\mathrm{KJ}$ herrühren.

\section{Zusammenfassung.}

Überblicken wir noch einmal das, was aus den vorstehenden Untersuchungen über die Beständigkeit der festen Polyjodide der Alkalien folgt, so finden wir, wenn wir zunächst die Trijodide nach ihren aufsteigenden Jodtensionen ordnen, die Reihenfolge:

$$
\mathrm{CsJ}_{3}=0.0033, \mathrm{RbJ}_{3}=0.0256, \mathrm{NH}_{4} J_{3}=0.053,
$$

und jedenfalls Kalium-, Natrium- und Lithium-Trijodid sich hier anschlielisend, da sie bei $25^{\circ}$ nur deshalb nicht existieren können, weil ibre Tensionen grölser sind als die des reinen Jods.

Die gegenseitige Reihenfolge dieser drei hypothetischen Trijodide lälst sich vorläufig nicht feststellen. Einen Anhalt über deren Abstufung erbält man jedoch durch die Tatsache, dafs Kalium bei $25^{\circ}$ ein anderes Polyjodid $\mathrm{KJ}_{7}$ bildet, während bei $\mathrm{Na}$ und $\mathrm{Li}$ auch dies nicht der Fall ist.

Ordnet man aiso die Alkalien nach der Tendenz zur Polyjodidbildung schlechthin, so ergibt sich die Reihenfolge:

${ }^{1}$ C. anorg. Chem. 2, 255. 


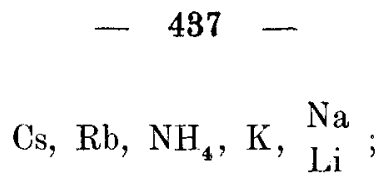

also etwa so, wie die Löslichkeitsreihenfolge der Chloroplatinate', Alaune, Chlorate, Nitrate und anderer schwer löslicher Salze und entsprechend ihrer Ordnung im periodischen System.

Biltz und WiLke-DörRfurt ${ }^{2}$, die ähnliche Erfahrungen bei der Untersuchung der Polysulfide machen, äufsern sich bei den entsprechenden Überlegungen folgendermafsen: „Man kann aus der Beständigkeit jener negativen Komplexe auf eine ähnliche individuelle Beeinflussung der diesen zugrunde liegenden einfachen negativen Bestandteile $\mathrm{S}$ resp. J durch die Nachbarschaft des $\mathrm{Rb}$ - und Cs-Atomes schliefsen, wie das bei organischen Verbindungen so überaus häufig ist. Wenn hiernach durch $\mathrm{Cs}$ oder $\mathrm{Rb}$ dem Jod oder Schwefel eine gröfsere Additionsfähigkeit induziert wird, so ist dieser Vorgang wohl mit der Aufzwingung einer Ionisierungstendenz in Parallele, ja in innere Beziehung zu bringen, wie sie ABEGg und BoDLAENDER ${ }^{3}$ in ihrer bekannten Theorie annehmen."

An Stelle dieser "Induktionswirkung" der Kationen ist vielleicht folgende Betrachtungsweise vorzuziehen: Wie die Untersuchung der in wässeriger Lösung sich bildenden Polyjodide gezeigt hat, entsteht im wesentlichen aus $\mathrm{J}_{2}$ und $\mathrm{J}^{\prime}$ - Ionen das Ion $\mathrm{J}_{3}{ }^{\prime}$, daneben jedoch noch merkliche, wenn auch geringe Mengen höherer Poly. jodid-Anionen. Es darf als sicher angesehen werden, dafs in der homogenen wässerigen Lösung die Polyjodidbildung lediglich eine Angelegenheit des Anions ist, somit die Konzentrationen der Polyjodid-Ionen $J_{3}{ }^{\prime}, J_{5}{ }^{\prime}, J_{7}{ }^{\prime}, J_{9}{ }^{\prime}$ in äquivalenten Lösungen der verschiedenen Alkalijodide wesentlich gleich sind, soweit nicht die wohl wenig verschiedenen Ionisationsverhältnisse geringfügige Unterschiede bedingen. Es ist aber keineswegs notwendig, dafs sich die Kombination des Kations gerade mit der in gröister Konzentration vorhandenen Anionenart als festes Salz abscheidet, vielmehr ist die Frage, welche Ionenkombination in fester Form austritt, lediglich

${ }^{1}$ Es lösen sich in $1000 \mathrm{~g}$ Wasser bei ca. $20^{\circ}$ in Äquivalenten etwa

\begin{tabular}{l|llll} 
& \multicolumn{1}{|c}{$\mathrm{Cs}$} & $\mathrm{Rb}$ & $\mathrm{K}$ & $\mathrm{NH}_{4}$ \\
\hline $1 / 2 \mathrm{PtCl}_{6}$ & 0.002 & 0.005 & 0.045 & 0.03 \\
$\mathrm{Al}_{6}\left(\mathrm{SO}_{4}\right)_{2}$ & 0.01 & 0.04 & 0.2 & 0.3 \\
$\mathrm{NO}_{3}$ & 1.2 & 3.6 & 3.2 & 2.5 \\
$\mathrm{ClO}_{3}$ & - & 0.3 & 0.57 & -
\end{tabular}

(berechnet nach Landoli-Bönsstein, 3. Aufl.)

${ }^{2}$ Ber, deutsch. ohem. Ges. 190อ̃, 123.

${ }^{3}$ Z. anorg. Chem. 20 (1899), 462. 
eine Angelegenheit der sehr individuellen jedesmaligen Löslichkeit, und es $\mathrm{mu}$ fs sogar ein höheres festes Polyjodid statt eines niedrigeren sich ausscheiden, wenn die Löslichkeit des höheren nur genügend viel geringer ist, als die des niederen. Dals sich bei Li und Na keine festen Polyjodide bilden, liegt dann daran, dafs die maximalen Konzentrationen der betreffenden Polyjodid-Anionen nicht genügen, um die betreffenden Löslichkeitsprodukte zu erreichen.

Da nun die höheren Polyjodide wie wir feststellten, $\mathrm{nm}$ so bevorzugter sich bilden, je weiter wir in der Reihe

$$
\mathrm{Li}, \mathrm{Na}, \mathrm{K}, \mathrm{NH}_{4}, \mathrm{Rb}, \mathrm{Cs}
$$

fortschreiten, so heifst das nichts anderes, als dafs in dieser selben Ordnung die Schwerlöslichkeit der höheren Polyjodide zunimmt. Diese Folgerung ist also nur ein weiterer Fall derselben Löslichkeitsreihen, wie sie oben für die Chloroplatinate, Alaune, Perchlorate angeführt wurden und wie sie, gemäls den Ausführungen von ABEGG und BodlatendeR ${ }^{1}$ als inverse Löslichkeitsreihen bei starken Anionen mit zunehmend starken Kationen sehr häufig festgestellt werden können. Hiernach sind die Polyjodid-Ionen als stark elektroaffin gekennzeichnet, und es ist bemerkenswert, dafs bei den einfachen Jodiden der Alkalimetalle eine solche inverse Löslichkeitsreihe zwar eben gerade erkennbar ${ }^{2}$, aber viel schwächer ausgeprägt ist als bei den Polyjodiden. Dal's diese durch Komplexbildung bedeutend stärkere Anionen geworden sind, steht gleichfalls im Einklang mit der Elektroaffinitätstheorie.

Die Frage, ob die Enneajodide wirklich die oberen Grenztypen darstellen, oder ob man nicht etwa durch Potentialsteigerung des Jods noch höhere Polyjodide erreichen könnte, ist wohl im ersteren Sinne zu entscheiden; denn die Erfahrung hätte sonst wohl schon höhere Typen kennen gelehrt, wo, wie bei Cs oder den Alkaloiden, deren Tendenz zur Polyjodidbildung sehr grols ist. Der Grenztypus wird offenbar durch die Valenzen des Jods bestimmt.

'1. c.; Am. Chem. Journ. 28 (1902), 222.

2 Für ca. $15^{n}$ ergeben sich die Löslichkeiten:

$$
\begin{array}{cccccc}
\mathrm{Li} & \mathrm{Na} & \mathrm{K} & \mathrm{NH}_{4} & \mathrm{Rb} & \mathrm{Cs} \\
\hline 12.3 & 11.5 & 8.4 & 11.5 & \mathbf{7 . 1} & 2.7
\end{array},
$$

$\mathrm{Mol}$ auf $1000 \mathrm{~g} \mathrm{H}_{2} \mathrm{O}$; also abgesehen vom $\mathrm{Cs}$ annähernd von gleicher Gröfse. Breslau, Abtlg. f. anorg. u. phys. Chemie d. Chem. Unit.Laboratorium. Bei der Redaktion eingegangen am 25. Juli 1906. 